

\title{
Scanning Transmission X-Ray Microscopy of Unaltered Biological Specimens
}

\author{
Nasif Iskander \\ Senior Honors Thesis \\ Lawrence Berkeley Laboratory \\ University of California \\ and \\ Department of Physics \\ University of California, Berkeley \\ Berkeley, California 94720
}

May 1987

\section{DISCLAIMER}

This report was prepared as an acoount of work sponsored by an agency of the United States Government. Neither the United Slates Guvernment nor any agency thereof, nor any of their employees, makes any warranty, express or implied, or ussumes any legal liubility or responsibility for the accuracy. completeness, or usefuiness of any information, appurtius, product, of process disclosed, of represents that its use would nol infringe privately uwned rights. Relerence herein to any specific commercial product, process, ur scrvice by trade name. rademark. manufacturer, of olherwise does not necessafily constitute of imply its cfidorsement, recommendalion, or favoring by the United States Government or any agency thereof. The views and opinions of authors expressed herein do nol necessarily state or reflect those of the United States Government or any agency thereor. 


\begin{abstract}
A scanning transmission $x$-ray microscope at the National Synchrotron Light Source was used to image fresh, wet biological specimens at 32 Angstroms, with resolution better than 750 Angstroms. A gold Fresnel zone plate (outer zone width 500 Angstroms) was used to focus the undulator radiation, and the sample was scanned through the spot. Absorption data was recorded digitally as a gridded array. The major accomplishment of the experiment was the demonstration of the ability to image biological samples in their natural state with high resolution and natural elemental contrast mechanisms. This was achieved through the design of a sample holder that maintains an aqueous environment for the sample, yet is transparent to $x$-rays at 32 Angstrons. The specimens used were isolated zymogen granules (approximately 1 micron diameter) from the pancreatic acinar cells of rats. The absorption data was corrrelated to protein concentration, and estimates of the protein concentrations within the granules were obrained. The daia also yields some information about the spatial organization of the protein in the granules, and our data is compared to models for the internal structure. The success of this experiment points toward future opportunities for dynamical studies on living systems
\end{abstract}




\section{Acknowledgements}

The work described here was made possible by the efforts and talents of many people. I would like to acknowledge the support, guidance and inspiration given to this project by $D r$. David Attwood of the Center for X-Ray Optics at LBL, and Professor Stephen Rothman of the Department of Physiology at UCSF. My sponsor in the Physics Department was Professor Steve Kahn. I wish to thank Harvey Rarback (NSLS) and Janos Kirz (SUNY Stony Brook) for giving us a share of the limited time available to use the microscope. The zone plates were manufactured at IBM by Yuli Vladimirsky of the Center for X-Ray Optics. Felping in the development and construction of the microscope were Harald Ade, Chris Jacobsen, and Ian McNulty of SUNY Stony Brook, Kate of NSLS, and Deming Shu of the Institute for High Energy Physics, Beijing. The biological specimens were prepared by Ken McQuaid of UCSF. The components of the wet cell were built by Dino Ciarlo of LLNL, and Phil Batson of the Center for X-Ray Optics.

This work was supported by an Air Force Office of Scientific Research contract with the Regents of the University of California for performance at the Lawrence Berkeley Laboratory, which is operated under DOE contract DE-ACO3$76 \mathrm{SF} 00098$. 


\section{Table of Contents}
Abstract
Acknowledgements
Table of Contents

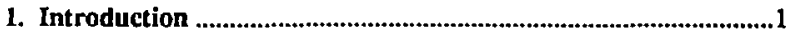
2. The Microscope

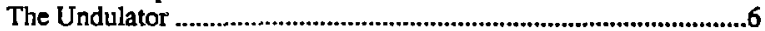
The Beamline ................................................................................9

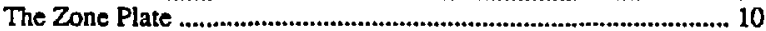

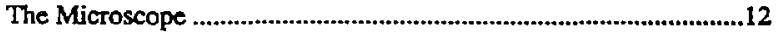

3. Soft X-Kay Absorption .......................................................... 15

4. The Specimens and Sample Holders

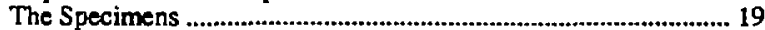

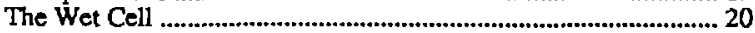

5. Data Analysis

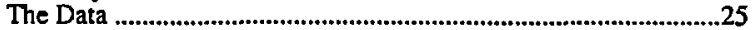

Calculating Protein Concentration ..................................................... 33

The Model of the Uniform Sphere .........................................................35

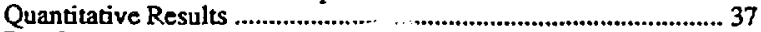

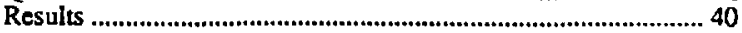

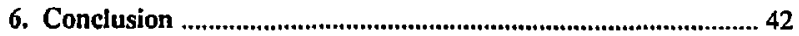

7. References 


\section{Introduction}

The two microscopy techniques currently relied upon by biologists are optical microscopy and electron microscopy. Each technique has significant drawbacks. Optical microscopy is limited in resolution by the wavelength of visible light. This means that features on scales below 1 micron cannot be studied. Electron microscopes have achieved atomic resolution, and have been the basic tool for studying subcellular morphology since the 1950's. There are, however, serious problems with studying biological systems with electron microscopy. First of all, the sample has to be placed in a vacuum. This means it has to be fixed and dehydrated. Secondly, there is no natural contrast between the lighter elements of interest to biology, and the specimen has to be selectively stained with heavy elements to arain an arificial contrast. Lastly, the specimens have to be exceedingly thin to allow partial transmission of the electron beam, so the specimens must be sectioned. Not only is the specimen unquestionably dead, but it is impossible to predict what sor of artifacts are being introduced during the preparation process.

Faced with these problems, researchers turned long ago to the possibility of using $x$-rays as a probe for high resolution biological imaging [1]. The shorter wavelengths mean the potential for resolurion on the molecular scale. The existance of inner shell transition edges for tiologically significant elements means natural contrast, even for light elements. The existance of the "water window" region of the soft $x$-ray spectrum, means that the imaging of wet specimens is a possibility. These properties make the soft $\mathrm{x}$-ray region ideal for the imaging of biological specimens, and makes high resolution dynamical studies a possibility. 
There are many problems with working in the soft $x$-ray region, however. It is extremely difficult to design and manufacture optical elements which work at these wavelengths. Almost all materials absorb $x$-rays strongly, and refractive optics is an impossibility. Much work has been done to perfect reflective imaging systems, but it is difficult to control abberations in this case. Also, good sources of $x$-radiation were difficult to find, and this fut a severe constraint on early research. It has been only recently, with the advent of synchrotron radiation, that high brightness $x$-ray sources have become available. Modem fabrication techniques allow the manufacture of diffractive $x$-ray optical elements, such as zone plates, to utilize this radiation effectively. In the new "renaissance" of $x$-ray optics, great strides are being made to overcome the technological hurdles and realize the potential of soft $\mathrm{x}$-ray imaging techniques.

Several schemes are currently being explored to develop useful $x$-ray microscopes. The scheme used in this experiement was scanning $x$-ray microscopy. Scanning microscopy yields numerical data as well as information about spatial relative aensities. It is also the method involving the lowest radiation dosage to the sample [2], an important consideration in planning dynamical studies. This purpose of this experiment was to demonstrate the potential that this technique has for the high resolution imaging of wet, unaltered biological materials, and the value of the numerical information recorded in the image.

Our experiement used a scanning ransmission $x$-ray microscope at the National Synchrotron Light Source at Brookhaven National Laboratory. The microscope was developed and is run by Harvey Rarback (NSLS) and Janos Kirz (SUNY Stony Brook). It was situated at 
beamline $X-17 T$ at NSLS. The microscope was designed to use soft $x$-rays in the $10-50$ angstrom range, and the source of the $x$-rays was an experimental undulator in the storage ring at beamline $X-17$. The radiation was spatially and chmmatically filtered to yield a partially coherent beam of monochromatic x-rays, which were focussed to a spot by a Fresnel zone plate. The zone plate used was made of gold on a silicon nitride substrate, with an outer zone width of 500 Angstroms [3]. The samples were held in a specimen holder of my own design, which allowed us to image them in their wet and unaltered state. The sample was scanned through the spot, in the focal plane, and a proportional counter measured the transmitted flux. The data was recorded digitally.

The data consists of a gridded array of count rates representing the flux transmitted through the specimen. The image is a two dimensional "numerical shadowgraph". If the flux incident on the specimen is known, the amount absorbed can be detemined. There is an easily calculable relationship between the fractional absorption through the sample and the mass density of the sample, if one knows the chemical composition of the specimen. The absorption is defined by:

$$
I_{\text {ransmitued }}=I_{\text {incident }} e^{-\mu \alpha}
$$

where $\mu$ is the mass absorption coeficient in units of $\mathrm{cm}^{2} / \mathrm{gm}$, and $\alpha$ is a measure of areal density of the absorbing material, in units of $\mathrm{gm} / \mathrm{cm}^{2}$. The values of $\mu$ are element and wavelength specific, and have been extensively tabulated [4]. There are two ways to extract information from this data. One method takes advantage of the sharp changes in the absorption at certain wavelengths, corresponding to energy thresholds for electron 
transitions. In between these absorption edges, the absorption as a function of wavelength is a slowly increasing relation. Thus, across two wavelengths relatively close together yet straddling an absorption edge, there will be a sharp contrast in the absorptive properties of the element with the edge, but almost no contrast for any other elements. If two images are taken, one on one side of an edge and another just on the other side of the edge, one can be subtracted from the other and the resulting subtracted image is an areal density map of the specific element with the absorption edge.

The situation is simpler in the case of our data. Our specimens basically contained only one type of substance, digestive enzymes. Using a generic recipe for the average animal protein, I calculated the mass absorption coefficient of "protein", and assumed that this was the only thing absorbing the $x$-rays in our images. Within the limitations of the noise in our data, the estimation of the mass absorption coefficients, and the uncertainties introduced with certain models I used in the analysis, I was able to calculate areal, and ultimately volume densities for protein storage in our specimens. I generated noi oniy spatial maps of concentration, but measurements of maximum and average concentrations of protein within the specimens, and these measurements compare favorably to previous measurements made with other techniques [5].

The experiment was performed in several runs over the course of the winter. My personal involvement with this experiment started in August of 1986, when I spent several weeks at NSLS helping to measure the undulator spectral output. I spent the fall semester designing the wet cell holders for our specimens and working with Professor Stephen Rothman (UCSF, Department of Physiology) on the biological aspects of the experiment. I went 
back to NSLS for three 5 to 10 day runs to take the data, in which I was responsible for the operation of the microscope and the beamline. I anlaysed the data at the Center for X-ray Optics at LBL during the spring semester of 1987. 


\section{The Microscope}

\section{THE UNDULATOR}

The radiation source at X-17T was a 10-pole magnetic undulator inserted in the $2.5 \mathrm{GeV}$ storage ring's electron beam path. It is broadly suneable in the soft $x$-ray region, and with the proper filtration can yield a large flux of coherent light. The undulator at X-17T was a test model of the 38 poie undulator now being constructed at $X-1$. The following is a discussion of how an undulator produces radiation.

A significant improvement in flux over synchrotron bending magnet radiation can be acheived through the use of insertion devices such as undulators. These magnetic structures are placed in the straight sections of the electron beam, and produce radiation along the beam axis. Undulators utilize a periodic magnet structure as depicted in figure 2.1 below. The electrons trayersing the device see alternating magnetic fields, and thus experience an alternating Lorentz force

$$
F=-e[Y \times B]
$$

perpendicular to their path. This causes them to follow a roughly sinusoidal path, wiggling along as shown in the figure.

The deflection parameter $\mathrm{K}$, is used to characterize undulators in terms of its magretic field strength. $K$ is defined by:

$$
K=\frac{e B a \lambda u}{2 \pi m o c}
$$




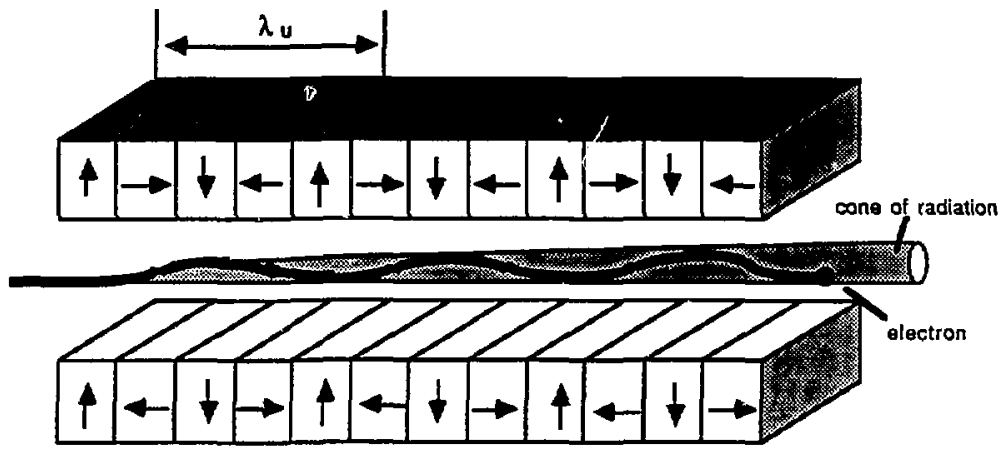

Figure 2.1: Schematic diagram of a permanent magnet undulator. The electron beam follows a sinusoidal parh in the horixontal plane, and radiates into a cone.

If $K \ll 1$ then the electron will experience acceleration only in the the direction perpendicular to its original pach, and the trajectory will be sinusoidal. If $K \geq t$, the electron's path is no longer close to a pure sinusoid, and there will be some radiation produced in harmonics of the fundamental wavelength.

In the electron's reference frame, the period of the undulator, $\lambda_{\mathrm{u}}$, appears Lorentz contracted to a wavelength $\lambda_{\mathrm{J}} / \gamma$. This is the period with which the elentron will oscillate. The oscillations will produce a radiation pattern like that shown in figure 2.2 below, when viewed in the electron's reference frame. In the laboratory frame, the light will be doppler 
shifted, and the wavelength on axis will be contracted to $\lambda_{v} /\left(2 \gamma^{2}\right)$. The pattern will have a narrowed frontal lobe, with half-angle $1 /\left(\gamma \mathrm{N}^{1 / 2}\right)$, as shown in figure 2.2. The fundamental wavelength and odd harmonics will be radiated into this pattern. The even harmonics will all appear off axis only, in two lobes with half angle $1 / \mathrm{f}$ as shown in figure 2.2 . The fundamental wavelength in the laboratory frame is given by

$$
\lambda_{\mathrm{rad}}=\frac{\lambda_{\omega}}{2 \gamma^{2}}\left(1+K^{2} / 2\right)
$$

For a real undulator, the electron beam has some finite emittance, and the observer will always be off-axis for some of the electrons. As a result, there will always be some occurrence of even order harmonics even on axis. For an undulator with a period on the order of centimeters, with $\gamma$ on the order of thousands, the fundamental wavelength is in the soft $x$-ray region.

Figure 2.2: Radiation patterns for electrons in an undulator. Furthest on the left is the radiation pattern for a sinusoidal oscillations in the electron's reference frame. In the center is this radiation pattern transformed to the laboratory frame. On the right is a diagram of the two-lobed partern in the laboratory frame into which the off-axis even harmonic radiation goes. [6]

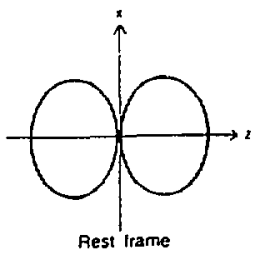

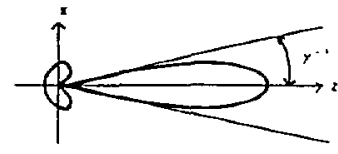

Laboratory Irame

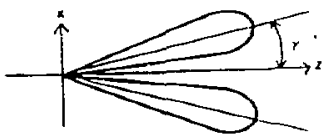

Laboratory Irame 


\section{THE BEAMLINE}

The X-17T undulator radiation was filtered to select the coherent portion of the beam for illumination of the zone plate. The "coherent" portion of the beam is diffraction limited radiation, with full transverse coherence, and a high degree of temporal coherence. The first element was a water cooled flat gold mirror which redirected the soft $\mathrm{x}$-rays into the branch -line. A 300 micron pinhole was then placed in the beam, to to select a spatially coherent portion of the beam. The monochromator was a toroidal grating, with the resolution set by the width of the source and the exit slit. This served as a source for the $\mathbf{5 0}$ micron pinhole, which finally selected a coherent portion of the beam as a source for the zone plate. Finally, right before the zone plate, the beam passed from vacuum into the air through a 1200 Angstrom silicon nitride window. Below is a schematic diagram showing the layout of major beamline components.

Figure 2.3: Schematic diagram (not to scale) of the major beamline components at $X-17 T$

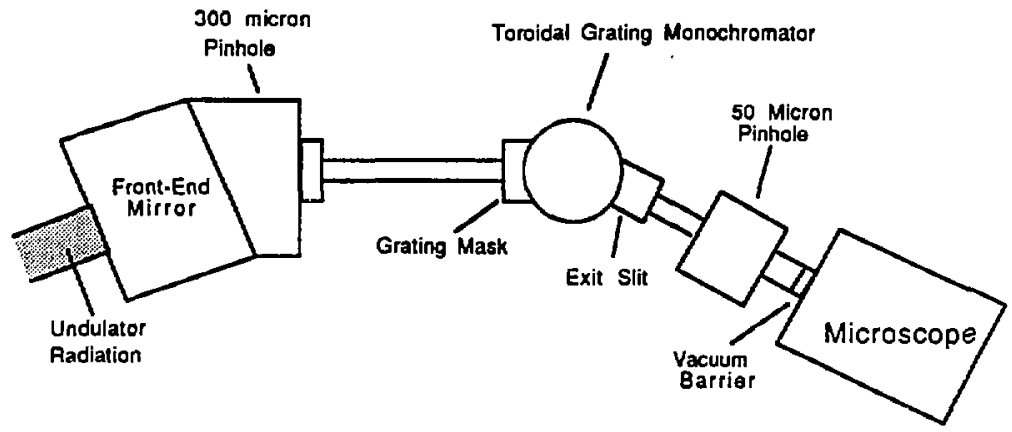




\section{THE ZONE PLATE}

The Fresnel zone plate, invented by Lord Rayleigh [7] gets its name from the fact that it focuses radaiation by blocking alternate Fresnel zones of a wavefront. It is essentially a circularly symmetric grating with radially varying spacing, and looks like the diagram below.

Figure 2.4: A Fresnel zone plate pattern [8]

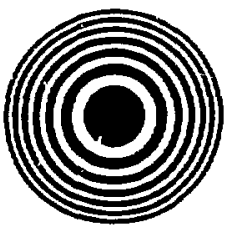

The easiest way to understand how a zone plate works is to think of it as a hologram of a point source. The zones on the surface of a spherical wavefront with radius $\rho$ defined by changes in phase of $\pi$ relative to an observation point a distance $R$ away are named Fresnel zones (see figure 2.5 below).

Figure 2.5: The definition of Fresnel zones

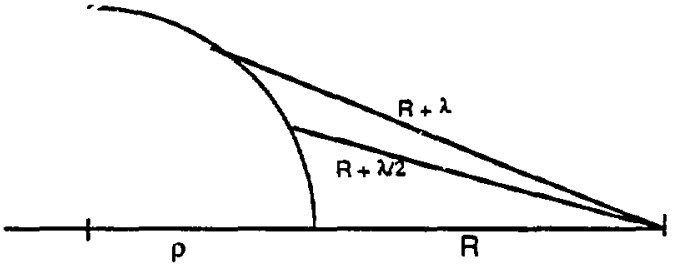


If this spherical wavefront were made to interfere with a plane wavefront, these zones would be alternately dark and light, due to destructive and constructive interference. A hologram recorded with this arrangement would look like figure 2.4 above, with the radius of the $\mathrm{m}^{\text {th }}$ zone given by:

$$
\mathrm{R}_{\mathrm{m}}^{2}=\mathrm{mr}_{0} \lambda
$$

When we illuminate the zone plate with coherent radiation, we are actually reconstructing the point source, thus focusing the radiation to a spot. Since it is a diffraction phenomenon, there are multiple orders reionstructed, and negative orders as well. In theory, about 10 percent of the incident intensity is diffracted into the first order focus. The angles of the reconstruction are dependant on wavelength, and thus the zone plate exhibits a high degree of chromatic aberration. The focal length $f$ is given by:

$$
f=\frac{4 \mathrm{~N}(\Delta r)^{2}}{\lambda}
$$

where $\mathrm{N}$ is the number of zones and $\Delta \mathrm{r}$ is the outermost zone width.

The zone plate is a high resolution focussing element. The size of the diffraction-limited spot is govemed by the width of the outermost zone, and is approximately $1.22 \Delta \mathrm{r}$. This defines the resolution of the zone plate using Rayleigh's criterion. The kayleigh range, or depth of focus, is given by

$$
\Delta Z=2 \Delta r^{2} / \lambda
$$

The zone plate that was used in this experiment was manufactured at IBM by Yuli 
Vladimirsky of the Center for X-Ray Optics, LBL. It had an outermost zone width of 500 Angstroms, and was made out of gold (1200 Angstroms thick) supported on a silicon nitride substrate (1000 Angstroms). The central zones were apodized io block the zero order transmission that would ootherwise contaminate the first-onder focus. At a wavelength of 32 Angstroms, the focal length of the zone plate was about $1 \mathrm{~mm}$, with a depth of focus of about 1 micron. The theoretical resolution was about 600 Angstroms, and the resolution was measured to be less than 750 Angstroms.

\section{THE MICROSCOPE}

The zone plate was used as a focusing elernent for our scanning microscopy. A schematic diagram of the microscope is displayed in figure 2.6 on the following page. A collimator was placed as shown in the figure, to eliminate unwanted orders diffracted by the zone plate. It also worked in conjunction with the central stop on the zone plate to eliminate zero-order contamination.

The specimen was mounted on the stage, and the zone plate was moved to place the focal spot in the specimen plane. The specimen could be sighted through an optical telescope. and coarsely (to 1 micron) aligned in the microscope's field of view using stepping motors controlled from a trak-ball. The fine motion for scanning the specimen during the exposure was provided by $X$ and $Y$ piezoelectric transducers under computer control. The $X$ and $Y$ positions of the stage were measured to within 300 Angstroms by a pair of fractional fringe HeNe laser interferometers. The stage was scanned smoothly, with dwell times at each pixel on the order of microseconds. We were able to make whole scans in a matter of minutes. 
The flux tränsmitted by the specimen was counted by a gas-flow proportional counter, and the counts from higher energy photons that came through as harmonics of the desired light were filtered out with an electronic discriminator. The data was recorded by the computer driving the scan. 


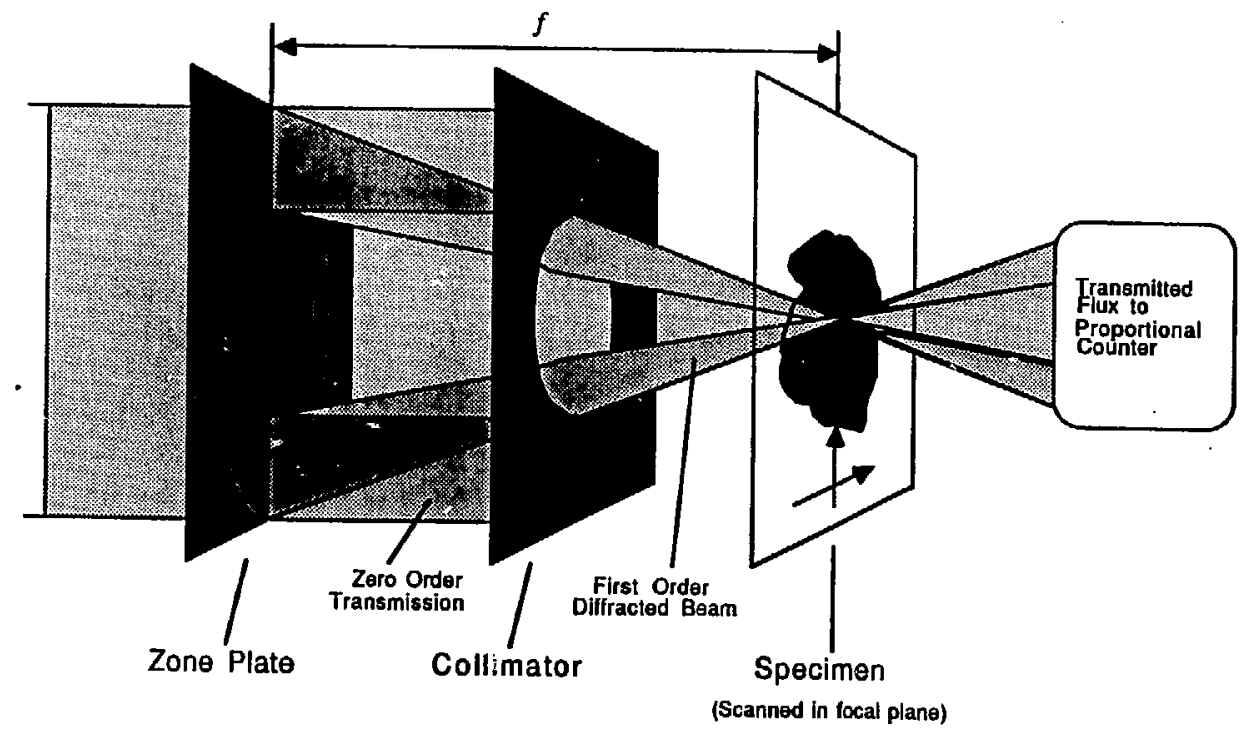

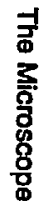

Figure 2.6: Schematic diagram of the microscope (not to scale) 


\section{Soft X-ray Absorption}

The beamline at X-17T was designed to operate in the 10-50 Angstrom range of soft $\mathrm{X}$-rays. This region is particularly interesting for work with biological materials, since this is the range of photon energies containing the $\mathrm{K}$-edge absorption features of many of the biologically important elements.

In this region, photons are absorbed primarily by bound electron transitions. The probability that a photon will be transmitted without absorption is given by

$$
\mid I_{0}=e^{-\mu \alpha}
$$

where $\alpha$ is the areal density of the absorbing material, and $\mu$ is the mass absorption coefficient, in units of area per mass. The mass absorption coefficient is a useful quantity for characterizing $x$-ray absorption, and it is dependant on both the wavelength of the incident

flux and the atomic number of the absorbing element. The relationship between $\mu$ and photon energy has sharp discontinuities due to the discrete electron transitions. These are the sharp discontinuities or "edges" referred to earlier. There is a specific energy required to movt, for example, an innermost shell (K-shell) electron to the next unoccupied state. If a photon has a lower energy than this threshold, it will not be absorbed by the K-shell electron. We see, then, at this threshold a sharp rise in the probability of photon absorption. The actual relation between mass absorption coefficient and photon energy looks something like figure 3.1 below. 
Figure 3.1: A typical plot of mass absorption coefficient versus wavelength

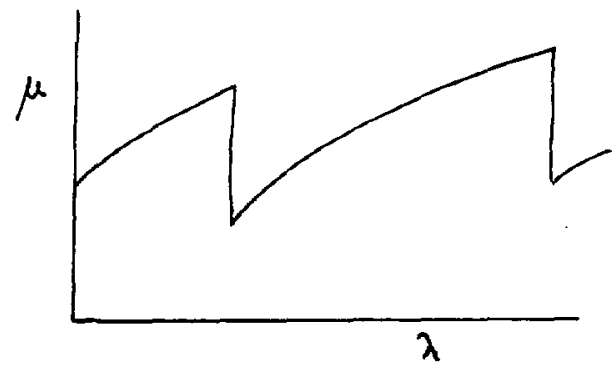

It is actually the K-shell transitions that are responsible for the absorption edges of

biologically important elements in the soft $x$-ray region. In figure 3.2 I have plotted $\mu$ versus wavelength for some of these elements.

For biological work, the region of interest is 23.6 Angstroms to 45.6 Angstroms. This is called the "water window", and is characterized by low photon absorption by water. This is because we are on the low energy side of the oxygen edge, so the $x$-rays can propagate more freely through the water. Looking at figure 3.3 will show that on the short wavelength (high energy) side of the oxygen K-edge, oxygen, carbon and nitrogen all have about the same absorptivity. It is only above 23.6 Angstroms that one can "see through" the water necessarily present in a living specimen, to detect the absorption specific to other substances within the sample. The long wavelength boundary of the water window is the carbon 
K-edge, at which point carton absorption drops below oxygen absorption, and once again the contrast is lost.

Figure 3.3: Mass absorption coefficient plotted versus wavelength for some elements of biological interest (C, N, O, Na, Cl, H)

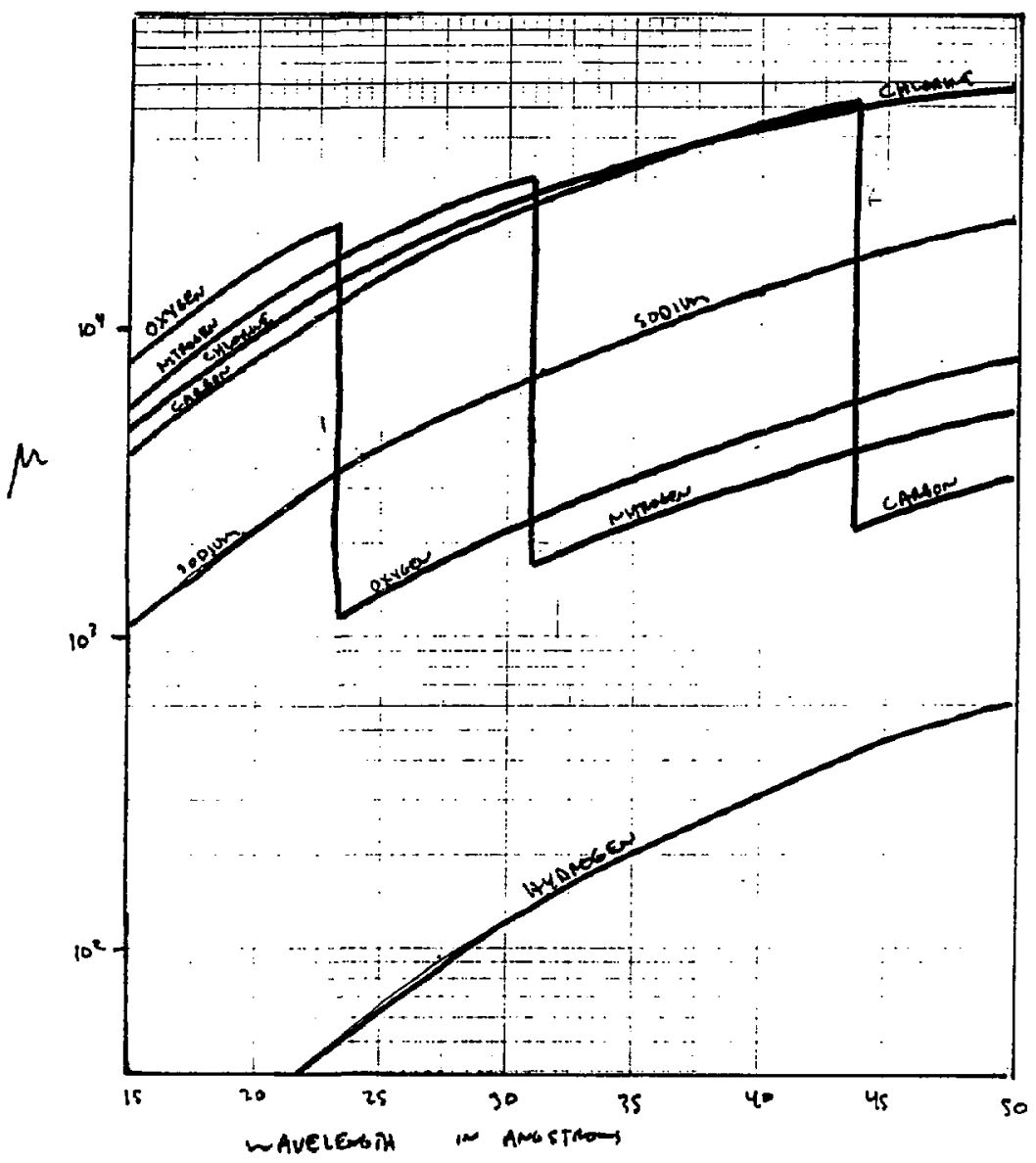


There was a further consideration in choosing a wavelength suitable for our experiment; that was nitrogen absorption. There were nitrogen-containing windows at various points along the beam path: the vacuum barrier, the zone plate substrate, and the proportional counter entrance window. More important than this, the microscope design incorporated a short beam path through air, to avoid having to place the sample in a vacuum. The atmospheric nitrogen would be highly absorbing in this region especially, so it was necessary to operate on the long wavelength side of the nitrogen edge, in the "nitrogen window", so to speak. This provides an inherent contrast between carbon and both air and water, which makes biological microscopy much easier. The wavelength we used was 32 Angstroms (indicated in figure 3.2) where the mass absorption coefficient for carbon is an order of magnitude higher than that for either nitrogen or oxygen. 


\section{The Specimens and Sample Holders}

\section{THE SPECIMENS}

The specimens we decided to concentrate our efforts on were subcellular components of pancreatic cells called zymogen granules. They are storage sites within the cell for the various digestive enzymes that the pancreas secretes. They have been studied extensively by electron microscopy, but are too small for their structure to be seen by optical microscopy. As a result, no work has ever been done which shows the internal structure of the zymogen granule in a chemically unaltered state.

The standard model of the granules is that they are spheres about 1 micron in diameter with uniform protein concentration throughout. Under the electron microscope, fixed, stained, dehydrated and sectioned, most of them appear as uniformly dark disks, with no visible internal variation or organization. Recent work indicates that some of them seem to have an internal reticulated network in which the preteins are stored. The ocurrence of "reticulated" grinules can be stimulated by making them lose some of their content before fixation [9]. It is unclear which aspects of the zymogen granules apparent morphology are artifacts introduced by the preparation process for electron microscopy. The images that we took are the Fist look at the natural intermal structure of the zymogen granule.

For our available methods, the most appropriate form for une granules to be in was isolated from the acinar cells in a sucrose suspension. Fresh tissue from the pancreas of a rat was ground in a morar and pestle, and by centrifugation the zymogen granules were seperated 
from the rest of the mixture. They could be kept, refrigerated, for several hours without spoiling. The sucrose solution in which they were suspended kept them in an environment similar to that of the cell from which they came. We also had hopes of imaging whole cells, and observing the distribution of granules within the cell. For this, suspensions of whole acinar cells were made in which they could be kept alive for several hours.

\section{THE WET CELL}

To image fresh, wet specimens, we needed a specimen holder that would hold the samples in a thin layer of water, and be transparent to the $x$-rays we were using. At: 31.5 Angstroms (just on the low absorption side of the nitrogen-edge), water is relatively transparent. A 1 micron thick layer of water will transmit $82 \%$ of the $x$-rays (see figure 4.1 ). However, as the water layer gets thicker, the transmissivity decreases quickly, and at 10 microns only $14 \%$ of the incident flux gets through. We set 10 microns as a reasonable upper limit to the thickness of the water layer in our wet cell.

We decided to use silicon nitride windows for the walls of our wet cell. Silicon nirride is a very strong material, that can be coated onto silicon wafers in very thin layers. The silicon substrate can be etched, and "windows" of silicon nitride can be made on the silicon frame. Silicon nitride has the added advantage of being transparent to $x$-rays with wavelengths above the nitrogen edge. A window of this sort was used at $X-1 T T$ as a vacuuni barrier. 
With a window area of 200 by 200 microns and a thickness of 1200 Angstroms it can hold atmospheric pressure [10]. The microscope stage had a magnetic mount for specimen holders, and these holders were thin rectangular stainless steel plates with a hole drilled near the top as a path for the $\mathrm{x}$-rays. We decided to design a wet cell that could be easily assembled, mounted on the specimen holder, and placed in the microscope in the standard fashion. There was an added constraint of clearance between the specimen in the focal plane of the zone plate and the collimator housing. This distance was approximately 200 mictons, and this had to be carefully watched to avoid damaging delicate elements of the microscope. The thickness between the specimen and the outer surface of our wet cell had to be about 100 microns to be easily mounted and used in the microscope.

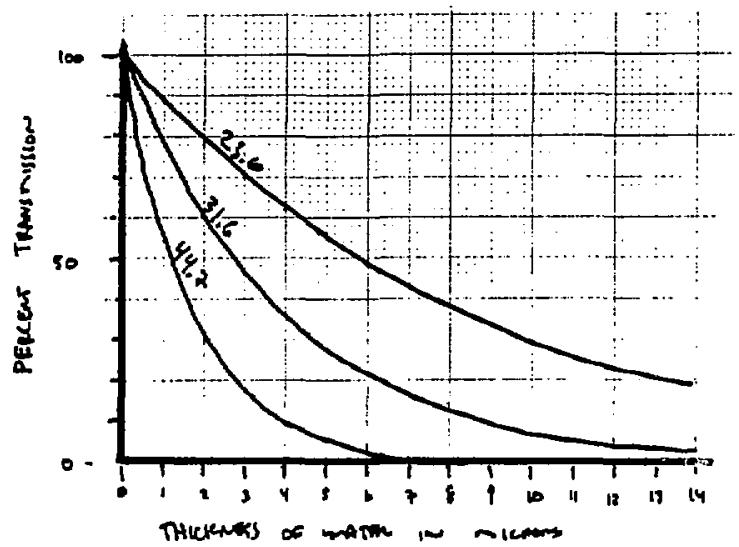

Figure 4.1: Percent transmission versus thickness of water layer for three wavelengths in water window: $23.6,31.6,44.7$ Angstroms 
We decided that if we placed two silicon nitride windows face to face, with the silicon nitride surfaces facing inward, we could compress a water layer between them as thin as we needed to. If we put a drop of the specimens in suspension in between the windows, our main problem would be keeping the windows far enough apart that they didn't damage our specimens. The granules are strong enough that they could hold the windows about 1 micron apart, and we would have a minimal thickness water layer for our specimens. The cells, however, are more fragile, and they were easily broken when we tried compressing the windows. We decided that we had to keep the windows about 10 microns apart to avoid rupturing the cells, but it couldn't be more than that wothout losing too much flux. Finding a 10 micron spacer wasn't easy, and I finally decided on 10 micron wire. It was pretty difficult to work with, and we ended up concentrating on the granules, which didn't need a spacer. Once the windows were compressed to our sarisfaction, we could seal the edges of the windows, and they would be held together by suction. Figure 4.2 below shows a cross section of a wet cell.

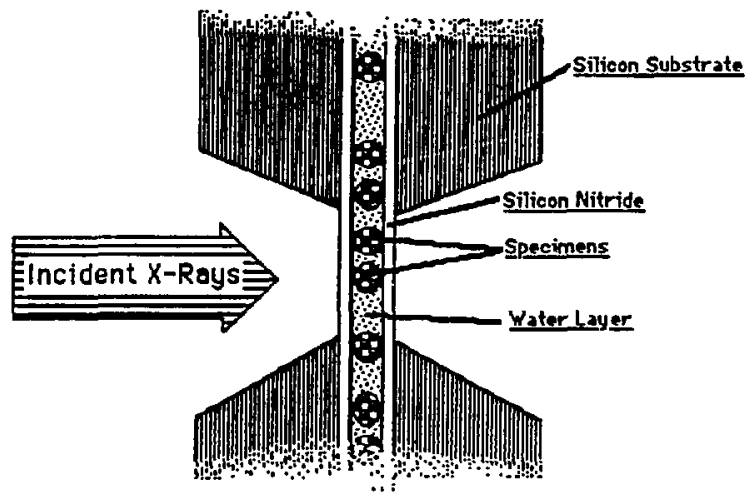

Figure 4.2 : Schematic diagram (not to scale) of a cross section through the window portion of our wet cell. 
My final design for the wet cell involved manufacturing very small windows ( 200 by 200 ) microns) in a large frame $(1.5$ by $2.0 \mathrm{~cm})$ so that they would be very strong, and have large enough frames to be easily handled. Dino Ciarlo at LLNL made the windows for us, on 4 mil (100 micron) silicon wafers, with silicon nitride layers 800 angstroms thick. To assemble the wet cell, we cemented one window silicon nitride side up onto the specimen holder. We placed a drop of the specimens (in suspension) onto the silicon nitride surface, and lowered the other window, silicon nitride side down, on top of the first one. I designed a small press that fits in the mechanical stage of a light microscope, and allows the experimenter to press the windows together by tuming a screw, while watching the window area through the microscope. When satisfied that the excess water has been expelled, and the thickness of the water in the wet cell is approximately the diameter of the granules (when the granules stop drifting), the experimenter can then remove the press from the microscope and seal the wet cell with a thin bead of cement. For the assembled wet cell to fit in the $x$-ray microscope, the cement had to be thin enough that the bead with which we sealed the windows didn't extend above the upper silicon surface. I found that nail polish thinned with acetone made a fine cement. Below is a photograph showing the various elements and stagti of preparation of the wet cell. 

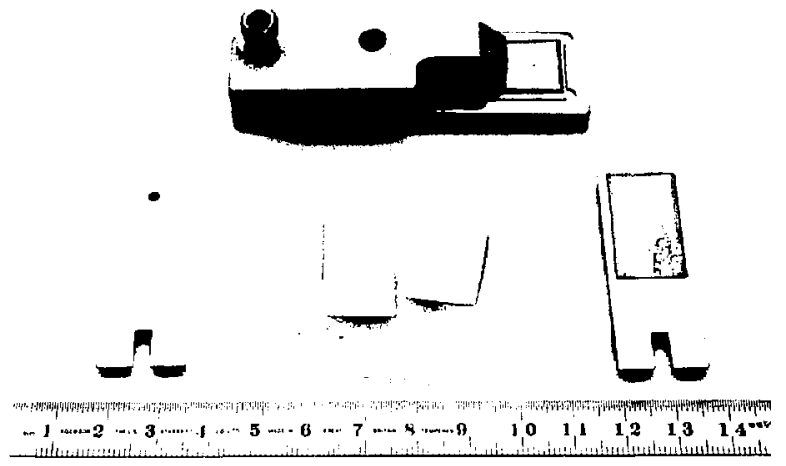

CBB 875-3813

Figure 4.3: Photograph of the various components of the wet cell and the different stages of assembly. At the front left is the microscope's sample holder. In the front center are the two silicon nitride windows that make up the walls of the wet cell. The window areas can just be made out about $1 / 4$ of the way down from the top. At the rear is the wet cell press, with a wet cell inside being sealed. At the front right is a sealed wet cell, ready to be inserted in the microscope. The scale in the foreground is in $\mathrm{cm}$. 


\section{Data Analysis}

\section{THE DATA}

The three most useful images we made were recorded on the $6^{\text {th }}$ and $7^{\text {th }}$ of February, 1987. 06FEB20 is an 80 by 80 pixel scan with a pixel size of 600 by 600 Angstroms. 07FTB14 and 07FEB 19 are both 60 by 60 pixel scans, with 600 by 600 Angstrom pixels. Figure 5.1 is a color representation of the data in the region of O6FEB20 containing a zymogen granule. The black represents a count rate of 49000 , and the white a count rate of 89000 .

Since we were looking for pixel to pixel spatial variation as an indication of changes in the absorbing stricture, noise in the data was a significant concem in the analysis of our images. The data sets of interest are scans of dark objects surrounded by "open" regions. We attempted to locate and image single granules whenever possible. These we identified as dense, roughly circular objects of approximately 1 micron diameter. The "open " areas around the objects are presumably relatively clear of organic material, and contain only a 1 micron thick water layer. Thus the variations in the count rates in these open areas are assumed to be representative of the noise in the incident radiation. Any noise in the incident radiation wil also be expressed as noise in the granule region of the scan. It is important to consider the variation due to noise when looking for variations in count rates as expressions of the internal structure of the granules.

There are several sources of noise in these open regions of the images. There is an inherent 


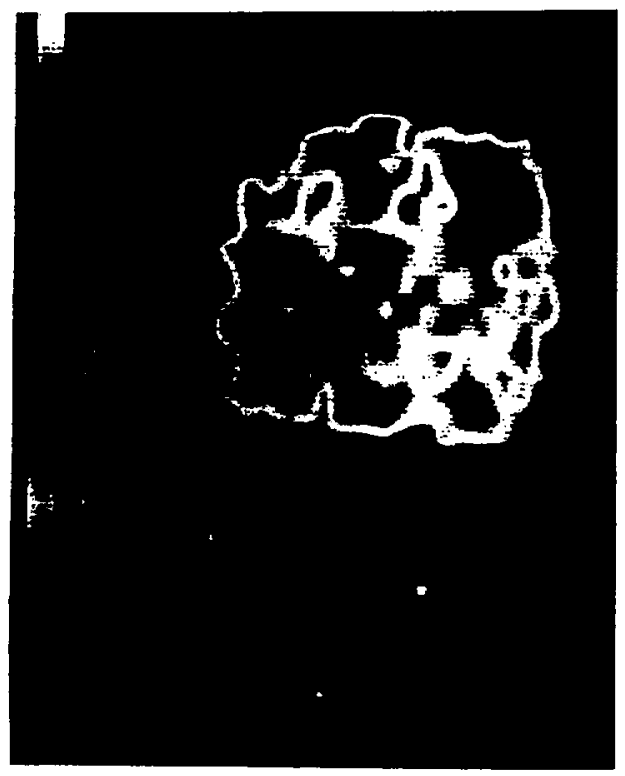

Figure 5.1: A color representation of the count rates in 06FEB20. Black corresponds to count rates of $49000 \mathrm{cps}$, and white to about $89000 \mathrm{cps}$ 
uncertainty in the count rates govemed by photon counting statistics. For a signal of $\mathrm{N}$ counts, this uncertainty is proportional to $\mathrm{N}^{-1 / 2}$. For the count rates in our images, the lowest of which are on the order of 50,000 counts per second, this correspond to less than $0.5 \%$ uncertainty. A more significant effect is the presence of beam instabilities. An unstable beam can not only change the count rate of the incident radiation, but change the monochromator source location, and thus the wavelength of the radiation selected from the beam. This can cause changes in the count rates through the specimen based on the changes in the mass absorption coefficients of the elements. This last effect is most problematic if the wavelength being used is near an absorption edge,. where the mass absorption coefficient for one element changes more dramatically with wavelength than for the rest. At 32 Angstroms, the nearest absorption edge is that of Nitrogen at 31.5 Angstroms. There are several Silicon Nitride windows along the beam path, including the two that make up the wet cell sample holder itself, and the portion of the beam path that is in air contains atmospheric nitrogen, making a total path length through nitrogen on the order of millimeters. These would become very strongly absorbing at 31.5 Angstroms, and would essentially extinguish the beam. We didn't experience any effects of this magnitude. There was a noticable periodic fluctuation in the beam intensity with a frequency of $12 \mathrm{~Hz}$, atributed to vibrational motion of the front-end mirror. We had planned to remove this regular noise from the images by Fourier analysis, but as yet haven't done that.

These have been real sources of noise in the incident beam photon count. There is another effect that is indistinguishable from real beam noise in the analysis that I performed. This is the presence of some organic material in what I have identified as open regions of the image. I have no way of seperating this information from the real noise, and will accept it as noise in 
my analysis. With this in mind, I have attempted to analyze the images first in terms of noise content. I plotted histograms of number of pixels with respect to count rates for each file. Figure 5.2 is a histogram of count rates for the image $06 \mathrm{FEB} 20$, in a 32 by 32 pixel area $I$ have identified as open. The distribulion looks Gaussian, centered at 73500 counts/sec, with a standard deviation of 3500 counts/sec, or approximately $5 \%$. In a histogram of a 32 by 32 pixel patch containing a granule, we see the same open area distribution super-imposed on a second distribution, presumably that of the count rates in the granule. If I could fit a Gaussian to the part of the distributrion corresponding to the open area of the image, I could extrapolate the fit and subtract it from the rest of the distribution, presumably leaving only the information about the granular region of the scan. Due to the large fluctuations in the "Gaussian" region of the histogram, resulting from the discrete sampling and averageing of the pixels, the quality of the fit would necessarily be poor, and the subracted histogram would have too much noise in it to be of value.

I did use the histograms to detemine a mean open area count rate, and a standard deviation for the count rates in the distribution for each image. On the following pages are histograms for the 32 by 32 pixel regions containing the granules in our 3 images. 


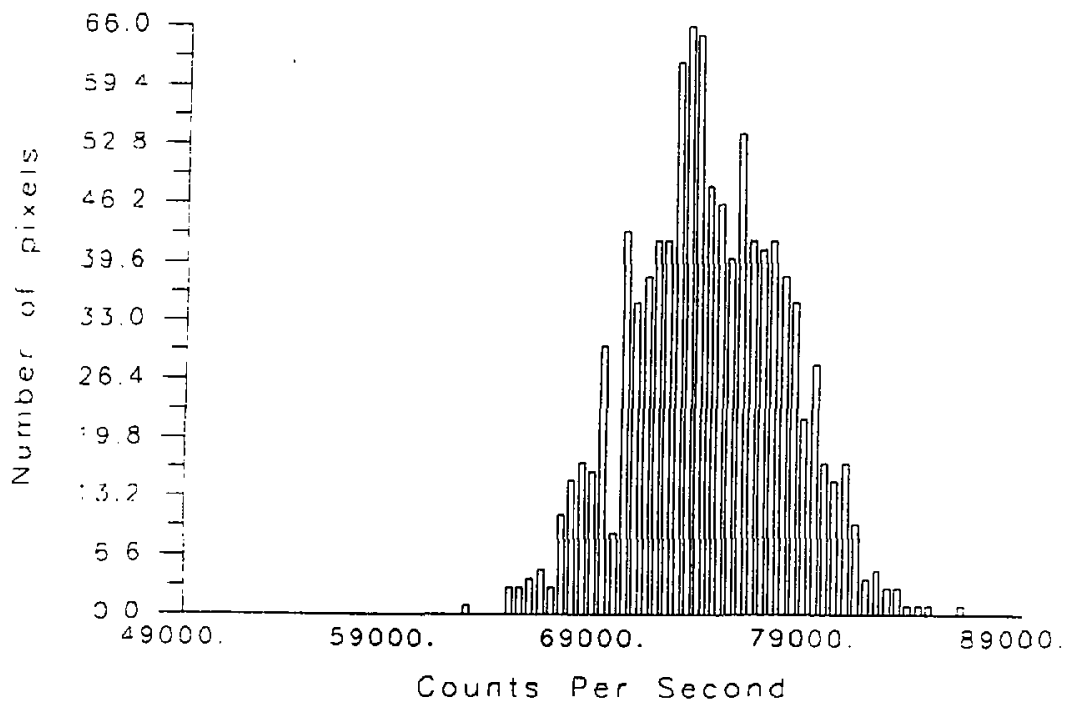

Figure 5.2: Histogram of an open 32 by 32 pixel patch of 06FEB20 


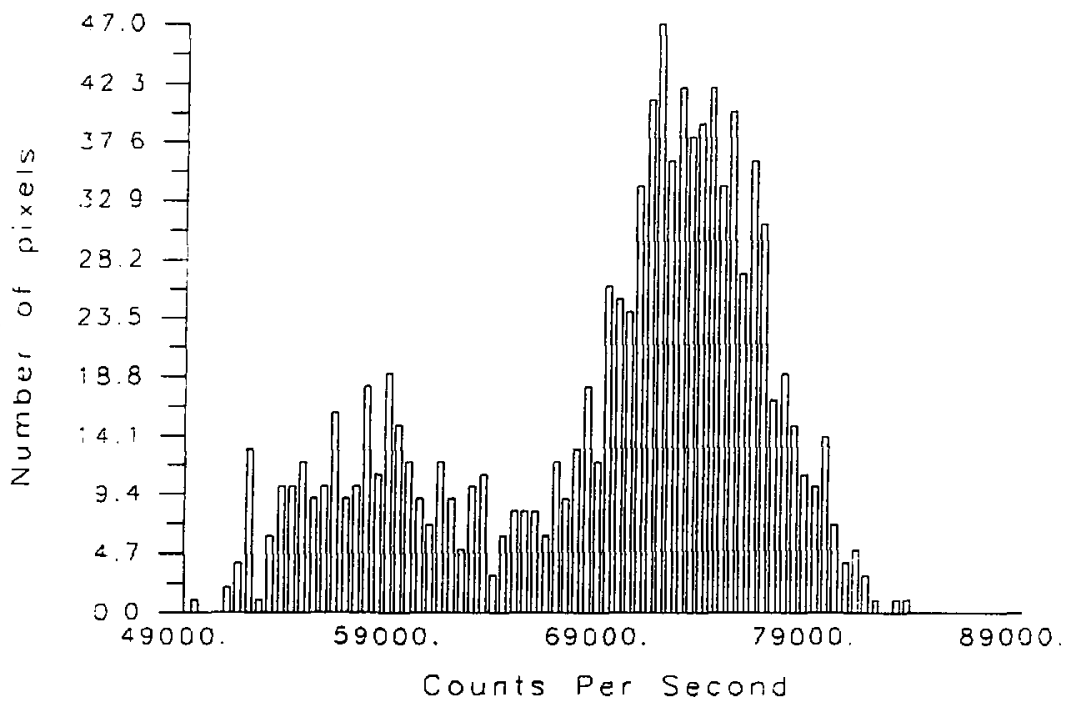

Figure 5.3: Histogram of 32 by 32 area of 06FEB20 that has a granule in it. 


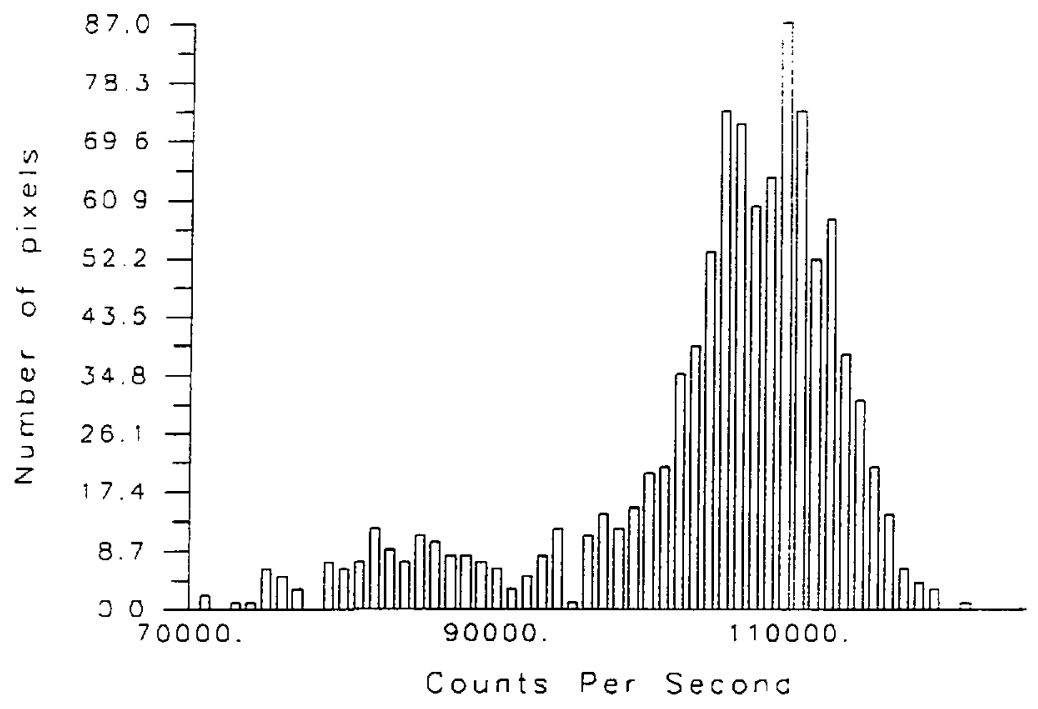

Figure 5.4: Histogram of a 32 by 32 patch of 07FEB 14 with a granule in it 


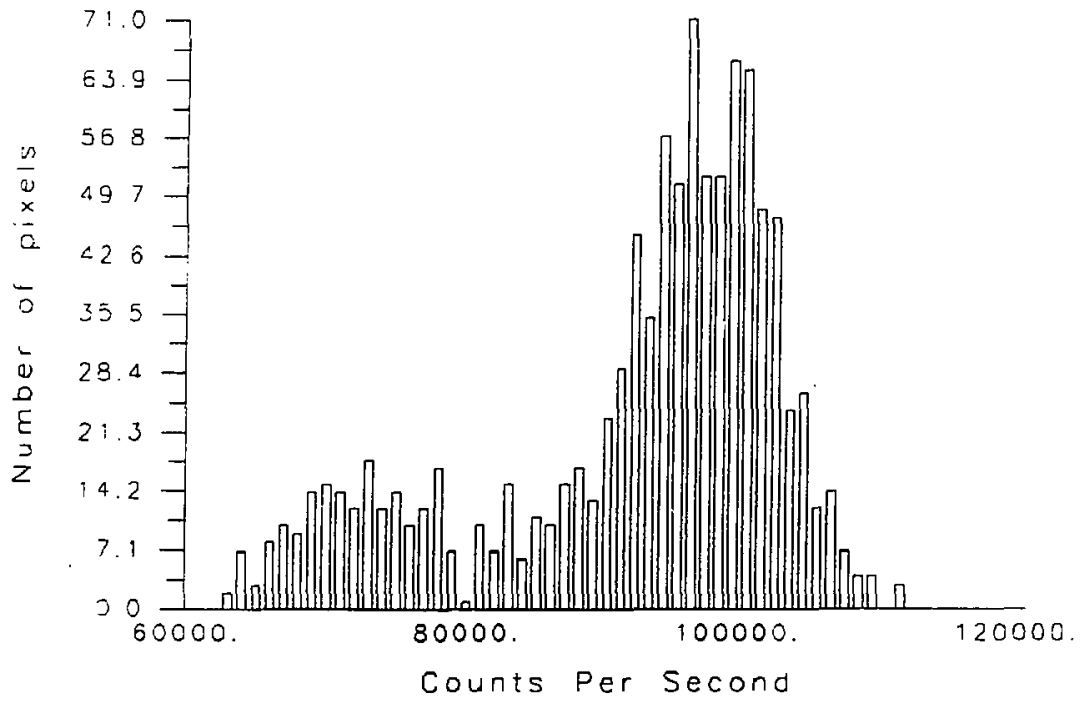

Figure 5.5: Histogram of a 32 by 32 pixel granule-containing region of 07FEB 19 


\section{CAlCulateng PROTEIN CONCENTRATION}

The first assumption I used to translate our absorption information into protein concentrations was that the only material in the granule that was absorbing the $x$-rays was protein [11]. There is a variety of proteins stored there, each with a different molecular composition. I used the chemical compositions of four of the proteins expected to be present,Trypsinogen, Chymotrypsinogen, Ribonuclease, and Carboxypeptidase [12], to calculate an average mass absorption coefficient for the granule material. The value I calculated for radiation at 32 Angstroms is $\mu=1.30 \times 10^{4} \pm 0.03\left[\mathrm{~cm}^{2} / \mathrm{g}\right]$.

In reality, the absorpion was taking place in both the protein and in the water of the granule, so that

$$
1=l_{0} e^{\left(-\mu_{1} \alpha\right)} e^{\left(-\mu_{2} \mid \rho\right)}
$$

where $\mu_{1}$ is the mass absorption of protein, $\alpha$ is the areal density of protein, $\mu_{2}$ is the mass absorption coefficient of water, $p$ is the volume density of water, and $l$ is the path length through the water. I was unable to determine what fraction of the path length through the wet cell was water, and what fraction was solid protein, and so assumed that the water absorption was a DC attenuation level across the whole image. If we assume that not more than $1 / 2$ of the path length is through protein at any given point, the maximum uncertainty resulting from this approximation is

$$
1-\operatorname{exp(-1/21.0\text {micron})}=0.1
$$




$$
\exp \left(-\mu_{2} \quad 0.5 \text { micron }\right)
$$

or about $10 \%$.This approximation allowed me to use the mean count rate in the open areas of the image as my incident count rate.

I also assumed that the thickness of the wet cell was determined by the thickness of thi granules (approximately 1 micton), and thus that the volume sampled by a pixel was 0.06 by 0.06 by $1 \mathrm{micron}^{3}$, or $3.6 \times 10^{-15} \mathrm{~cm}^{3}$. This allowed me to express my results in units of $\mathrm{mg} / \mathrm{ml}$, convenient units for comparison to previously reported measurements. At each pixel, I soived for the areal density $\alpha$ in the equation

$$
\alpha=\ln \left(/ / / I_{0}\right) / \mu
$$

and divided this value by the 1 micron thickness of the wet cell, expressing the result in $\mathrm{mg} / \mathrm{ml}$. I have plotted the results of these calculations in contour maps on the following pages. The uncertainty associated with these calculations is :

$$
\sigma(\alpha)=\sigma(l) 1 /(\mu l)
$$

These uncertainties are $39 \mathrm{mg} / \mathrm{ml}$ for 06 FEB20, $30 \mathrm{mg} / \mathrm{ml}$ for $07 F E B 14$, and $41 \mathrm{mg} / \mathrm{ml}$ for 07FEB 19. For this reason, I chose a contour interval of $50 \mathrm{mg} / \mathrm{ml}$ for the maps. I ignored negative values of concentration. 


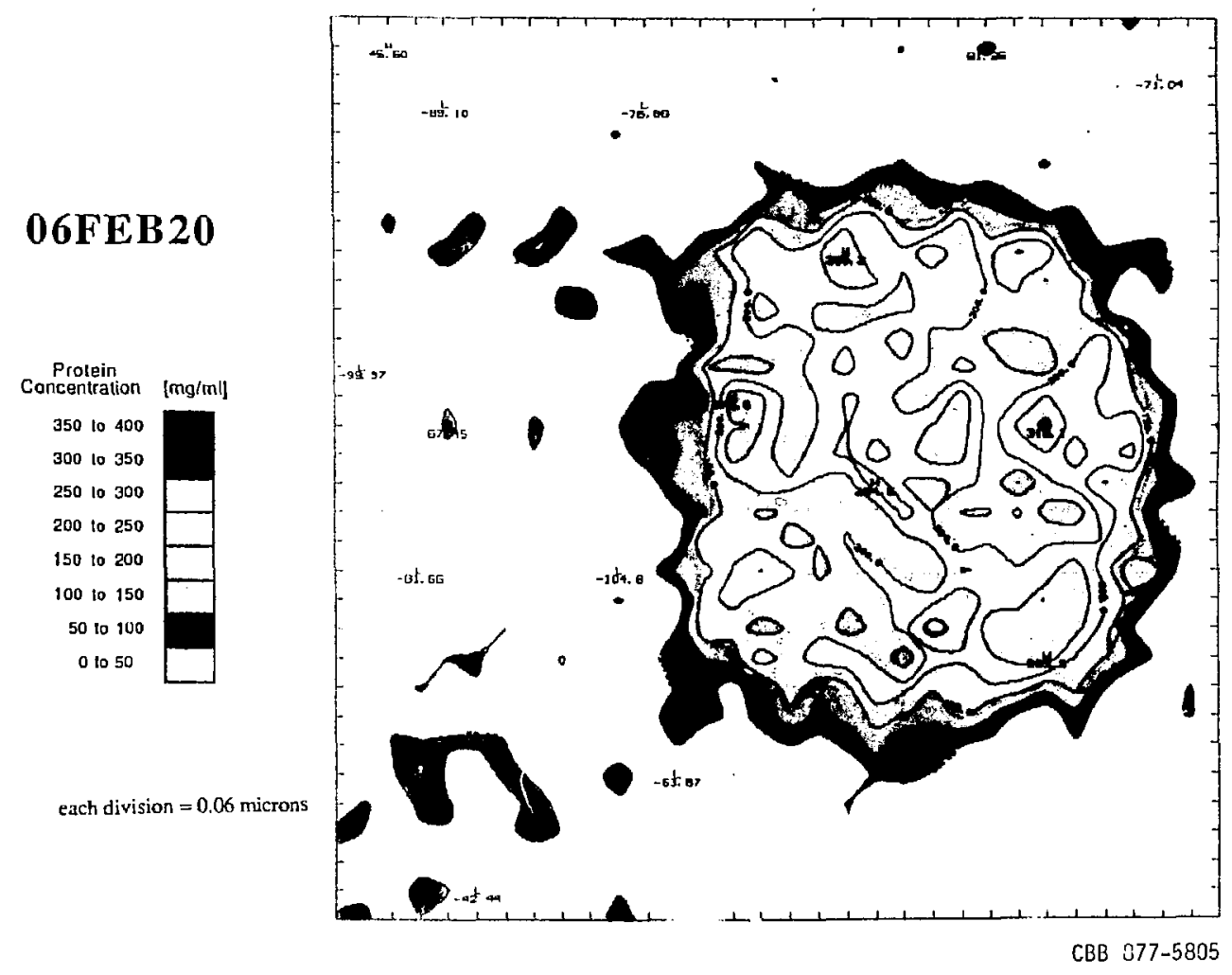




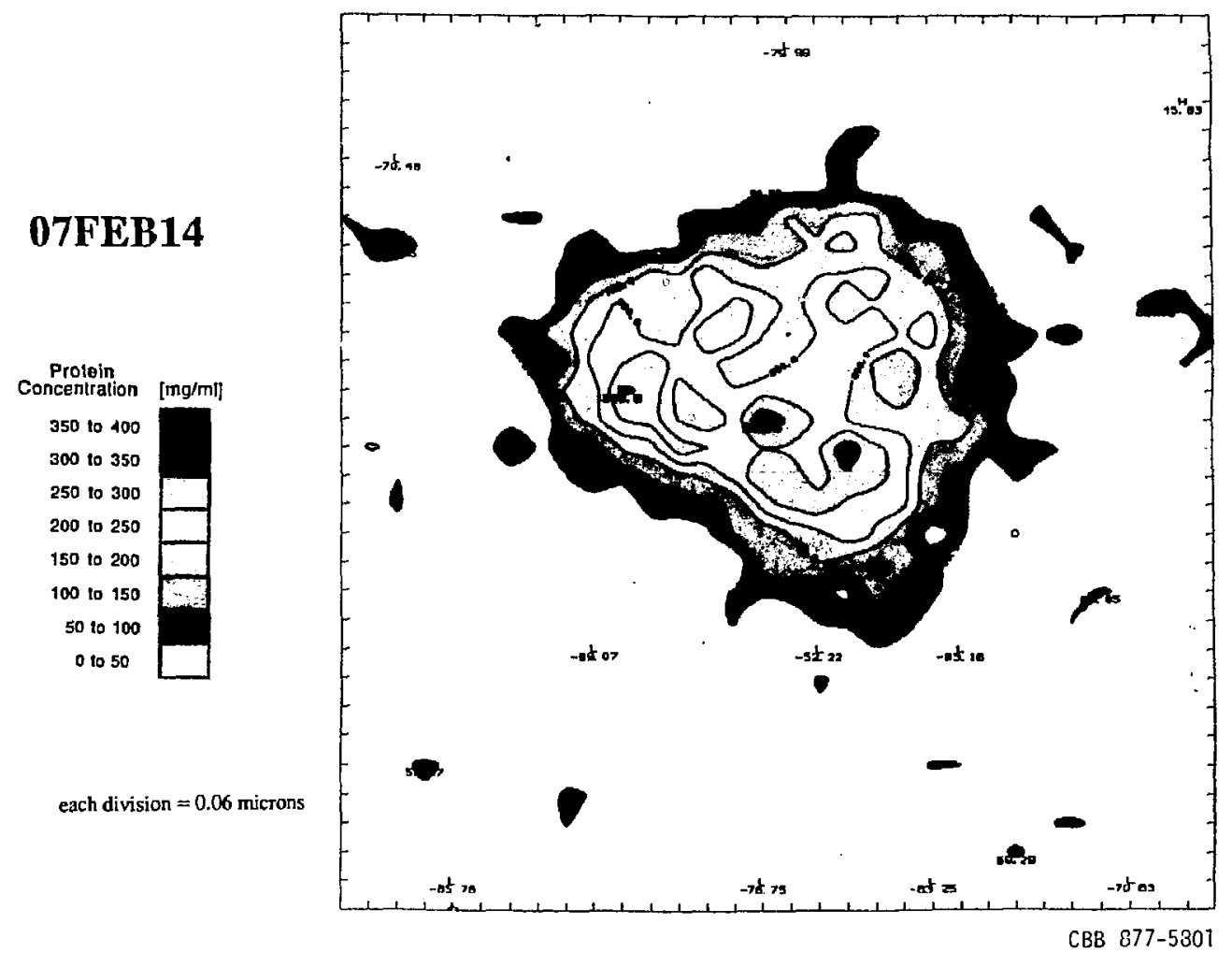




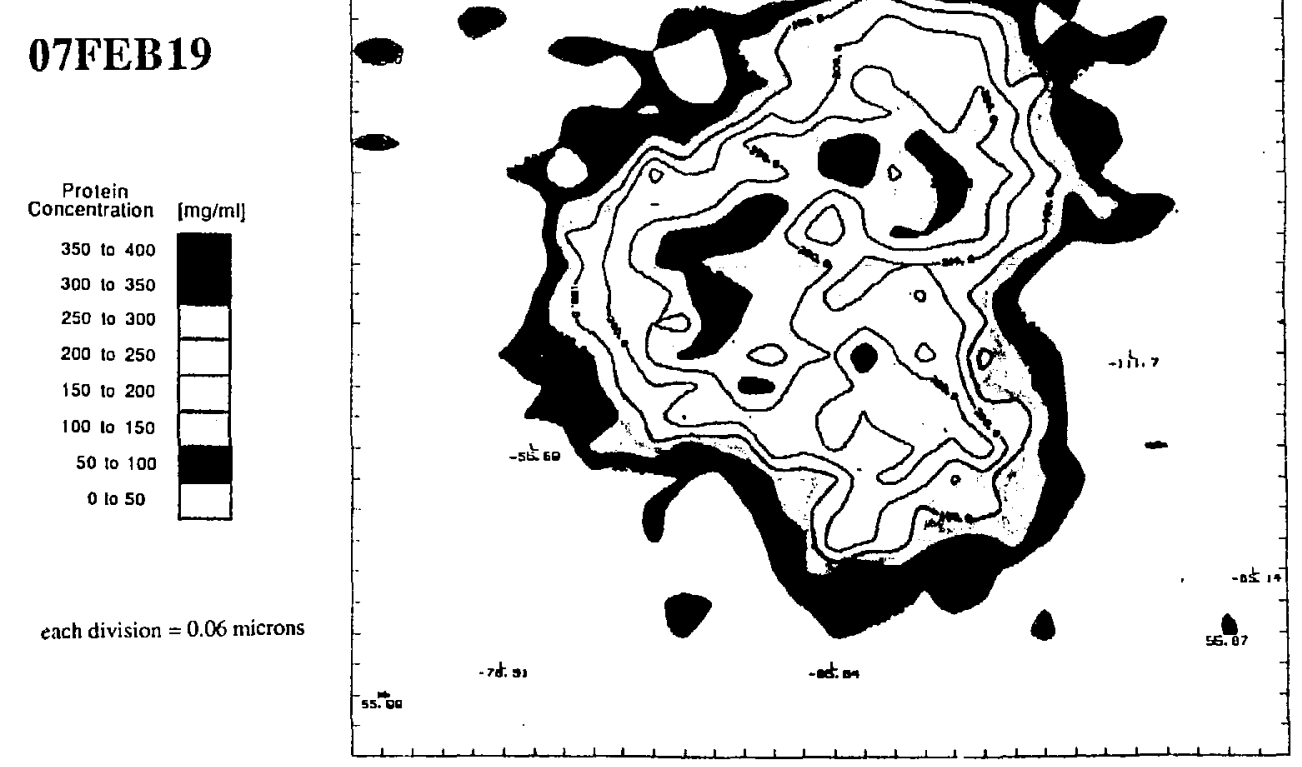




\section{THE MODEL OF THE UNIFORM SPHERE}

The primary motivation for imaging the granules was to obtain structural information. It was imporant, therefore, to know what features in the images could be artifacts of the discrete sampling or noise. Since we were interested in comparing the images to the standard model of the uniform sphere, I created an artificial data set that modeled the image of a uniform sphere.

The code that generated the data set was actually a simulation of the microscopy experiment. All of the parameters of the sphere such as radius (in pixels), density (in $\mathrm{mg} / \mathrm{ml}$ ), and mass absorption coefficient were entered along with the incident beam intensity, and the program simulated a 32 by 32 pixel scan. Each image pixel was broken down into 10 by 10 sites, and the mass of absorbing material was calculated for each. The results were averaged over the whole pixel, and this average was used to calculate the transmitted fraction of the incident beam at this location. The array of count rates was stored under the name of SPHERE in exactly the same format as the real images, so that it could be read and analyzed in exactly the same way as the real scans.

To simulate the effects of a noisy beam, I also generated a data set of the same model with random fluctuations applied to the incident beam intensity. I set the width of the fluctuations to $6.7 \%$, slightly higher than the standard deviations measured from the real images. I analysed this model, SPHERE_STAT, alongside the three real images, and in exactly the same fashion. Since I knew exactly what object this data set represented, I had a check against false conclusions that I might otherwise have drawn about the real granules. The 
contour maps of protein concentration that I calculated form SPHERE and SPHERE_STAT appear on the following pages.

Looking at the concentration map for SPHERE_STAT and comparing it to the three real images, we can see an interesting difference in the overall protein distribution. In the spherical model, all of the highest densities of protein occur, as we would expect, at the center of the object. This is not true for the real granules. In their case, most clearly for O6FEB20, the regions of highest density are closer to the edges of the granule. This suggests a very different structure than the uniform sphere model.

There is one unusual feature that the granules share with SPHERE_STAT, that is the apparently complicated edge shape. This is obviously an artifact since it appears in the model, and it must stem from the discrete sampling of the image in pixels, coupled with the statistical variations from pixel to pixel. 


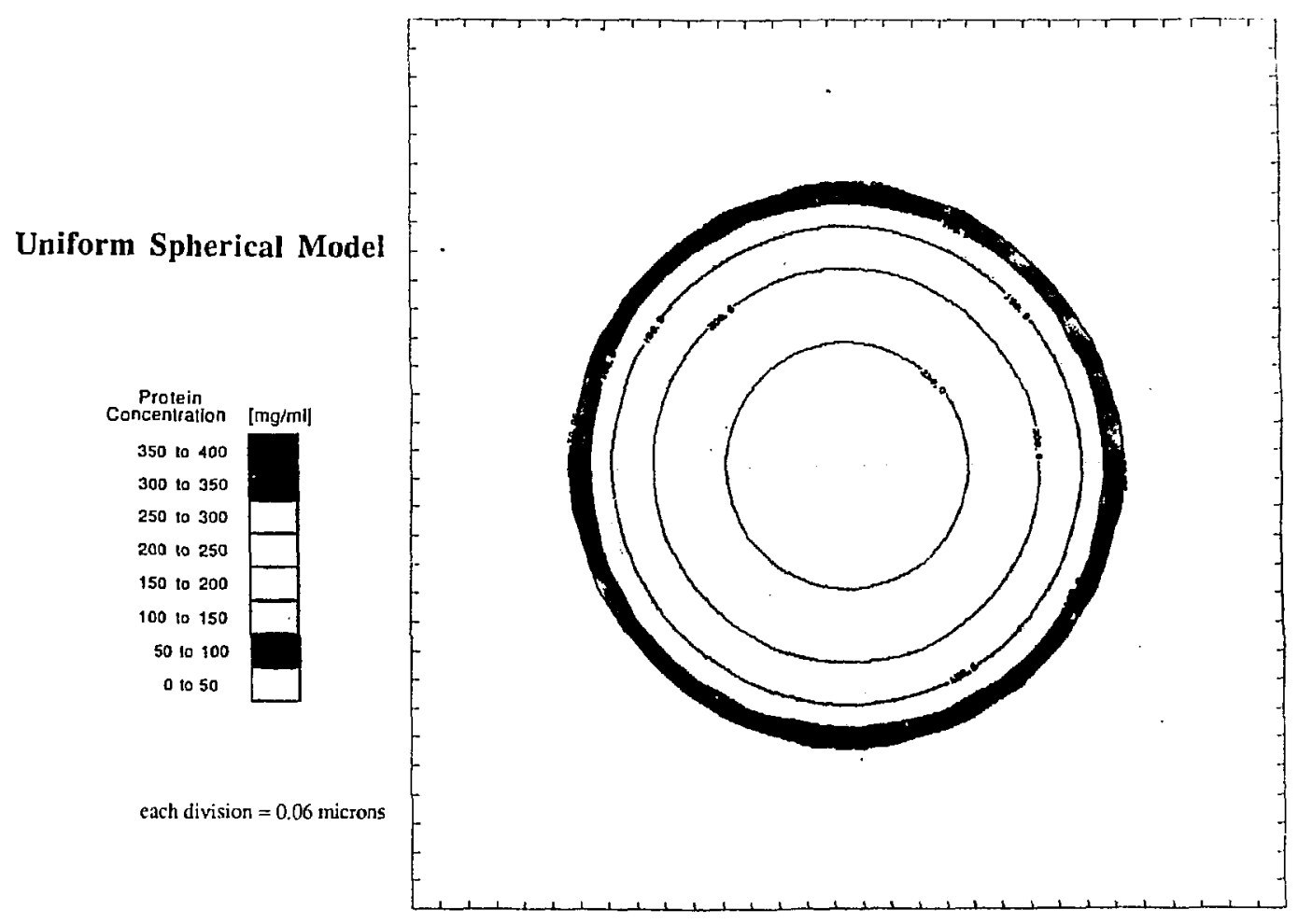

CBB 877-5799 
Uniform Spherical Model with Statistical

Fluctuations Applied

Protein

Concentration

350 to 400

300 to 350

250 to 300

200 to 250

150 to 200

100 to 150

50 to 100

0 to 50

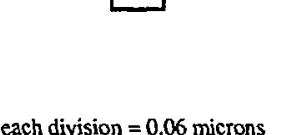

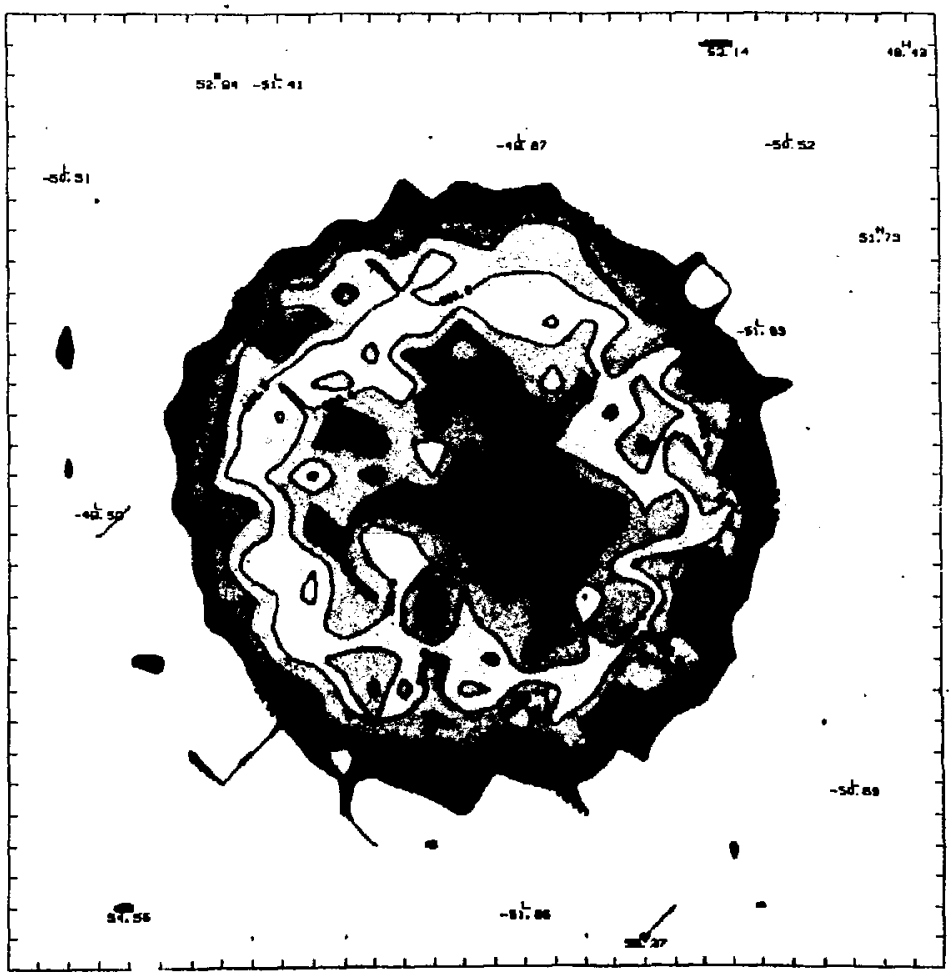

CEB 877-5807 


\section{QUANTITATIVE RESULTS}

Our data contains more than just spatial information about the relative density of our samples. We have numerical information from which we can calculate, within the uncertainties of my approximations and the noise in the data, absolute quantitative measuerments of protein concentration. These can then be compared to values obtained by different techniques [13].

1 wrote a program that integrates the total mass of stored protein over the images of the granules. It does this by adding up all of the calculated mass contained in each pixel column through the specimen, for pixels exhibiting concentrations above a pre-defined threshold. It also counts the number of pixels that it used in the calculation, and assumes that they were all in one circular region of the image. With this assumption, it calculates the radius of this circle, and assumes that this circle is the two dimensional projection of a spherical object. It calculates the volume of this sphere. Now it can divide the total integrated mass of the protein over the volume of the sphere that it fitted to the data, and obtain a value for the average internal concentration of protein in the sphere.

1 chose a threshold of $50 \mathrm{mg} / \mathrm{ml}$, for the same reason that $I$ used it for the contour intervals. The boundary of the granule for this scheme matches the boundary of the lowest contour line in the concentration maps. The results of the calculations are listed in the following table. 
TABLE 5.1

\begin{tabular}{|l|l|l|l|l|l|}
\hline Quantity & 06FEB20 & 07FEB14 & O7FEB19 & SPHERE_STAT & $\begin{array}{l}\text { Original } \\
\text { Parameters }\end{array}$ \\
\hline Radius (pixels) & 10.70 & 8.46 & 10.25 & 10.49 & 10.00 \\
\hline $\begin{array}{l}\text { Total mass of } \\
\text { protein }\left(\mathrm{mg} \times 10^{-10}\right)\end{array}$ & 2.06 & 1.18 & 2.0 & 2.32 & 2.09 \\
\hline Average conc. $(\mathrm{mg} / \mathrm{ml})$ & $248 \pm 39$ & $289 \pm 30$ & $280 \pm 41$ & $300 \pm 54$ & 274 \\
\hline
\end{tabular}

By comparing the results for SPHERE_STAT with the original input parameters, we can check the validity of the results obtained for the real granules. We can see that the total mass content of the sphere has increased. This is due to contamination by pixels with unusually low count rates in the open areas of the image. As a result, the apparent size of the object is also increased. All of the differences are well within the calculated uncertainty, however, and the results for the real granules have some value.

We can apply the fitted sphere to the data to estimate the upper and lower bounds on the internal concentration of protein within the zymogen granules. I drew lines through the center of each granule in the images, also passing through the points that looked like they represented the highest internal concentrations of protein. I then took the count rates for the pixels along these lines, and translated them into protein concentration, in the same way as was done for the contour maps. These then had to be translated into internal concentrations of protein.

This involved reducing the volume of the column sampled by each pixel to the porion of that column that was contained within the fitted sphere. This is shown diagrammatically in the 
figure below.

Figure 5.6: The relationship between concentration in the wet cell and internal concentration within the granules. On the left, the protein concentration is averaged over the entire hieght of the wet cell. On the right, the internal concentration is displayed, and the volume of the column has been adjusted to fit inside the sphere.

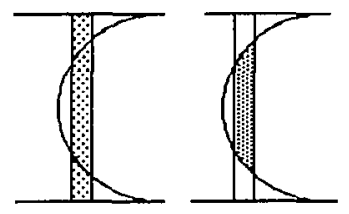

The figures on the following pages show the four images, 06FEB20, 07FEB14, 07FEB 19, and SPHERE_STAT with the two-dimensional projection of the fitted sphere (shown as a dashed circle), and the line profile that I used. Also indicated are the individual pixels that I used to represent the line. On the page immediately following each image, is a set of 3 graphs showing the profile of the image along the specified line. The first graph shows the count rate of each of the marked pixels. The second graph shows the protein concentration calculated for the entire thickness of the wet cell. The last graph shows the protein concentration adjusted to the fitted sphere, to show internal protein concentration for the granule. The maximum and minimum values of the internal concentration are tabulated below . 

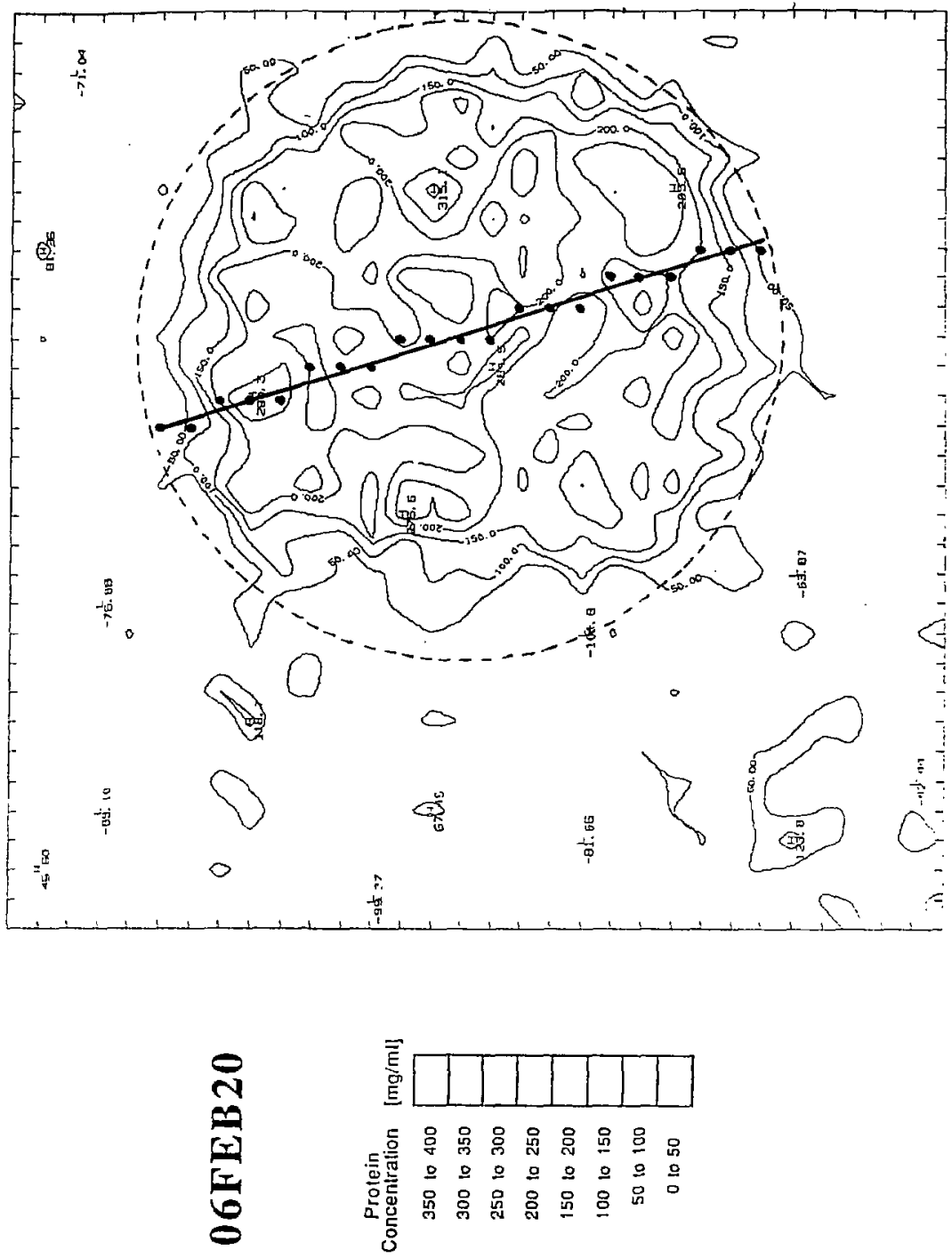

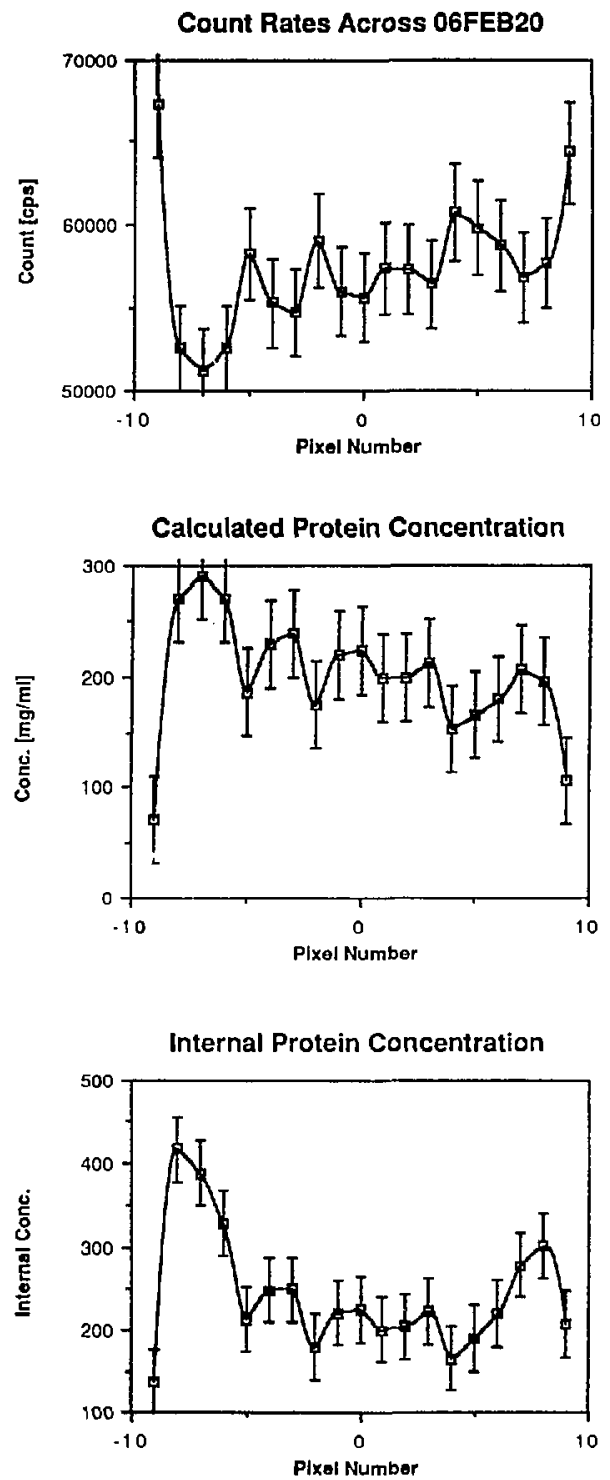


\section{FEB14}

Protein
Concentration $[\mathrm{mg} / \mathrm{m}$ ]
350 to 400
300 to 350
250 to 300
200 to 250
150 to 200
100 to 150
50 to 100
0 to 50

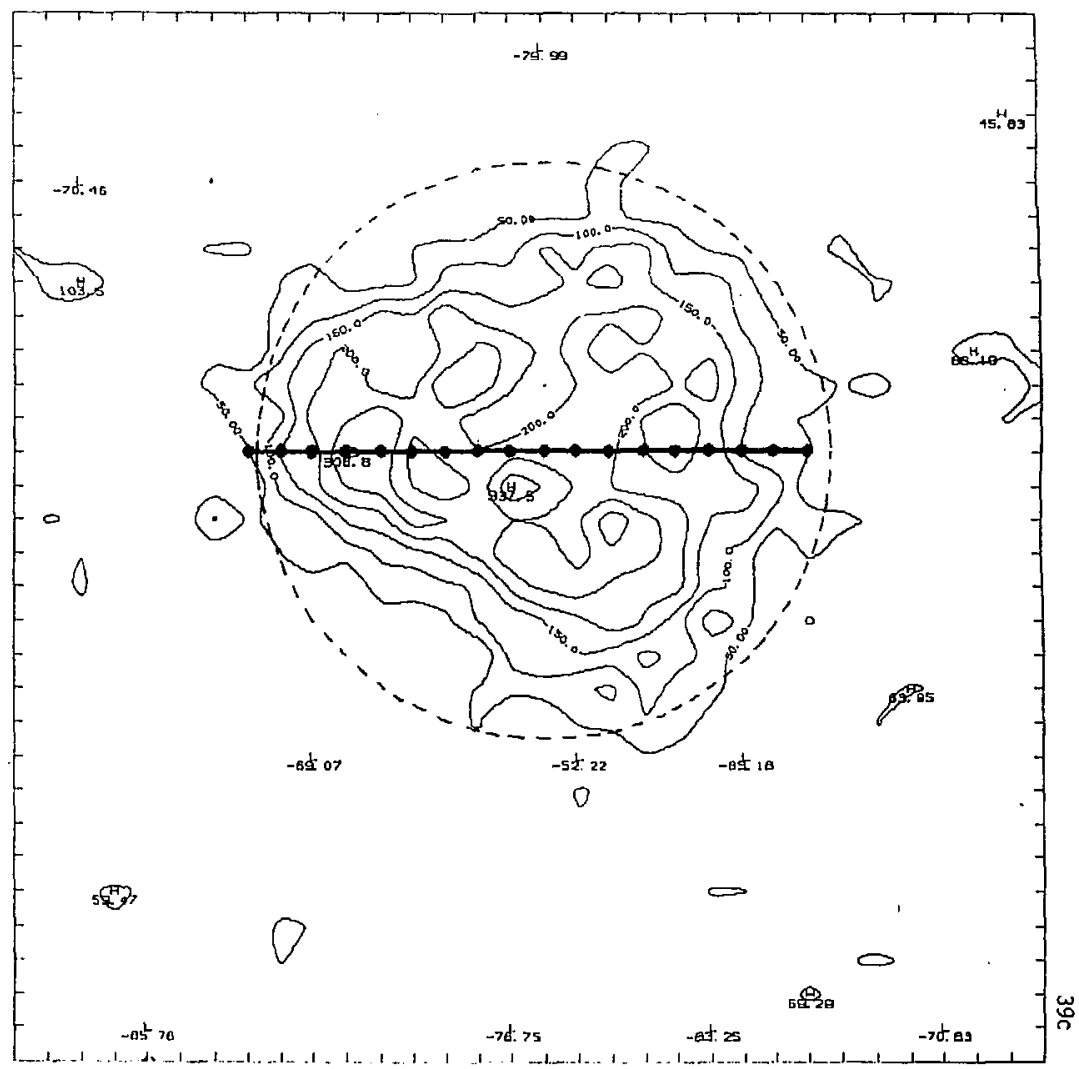



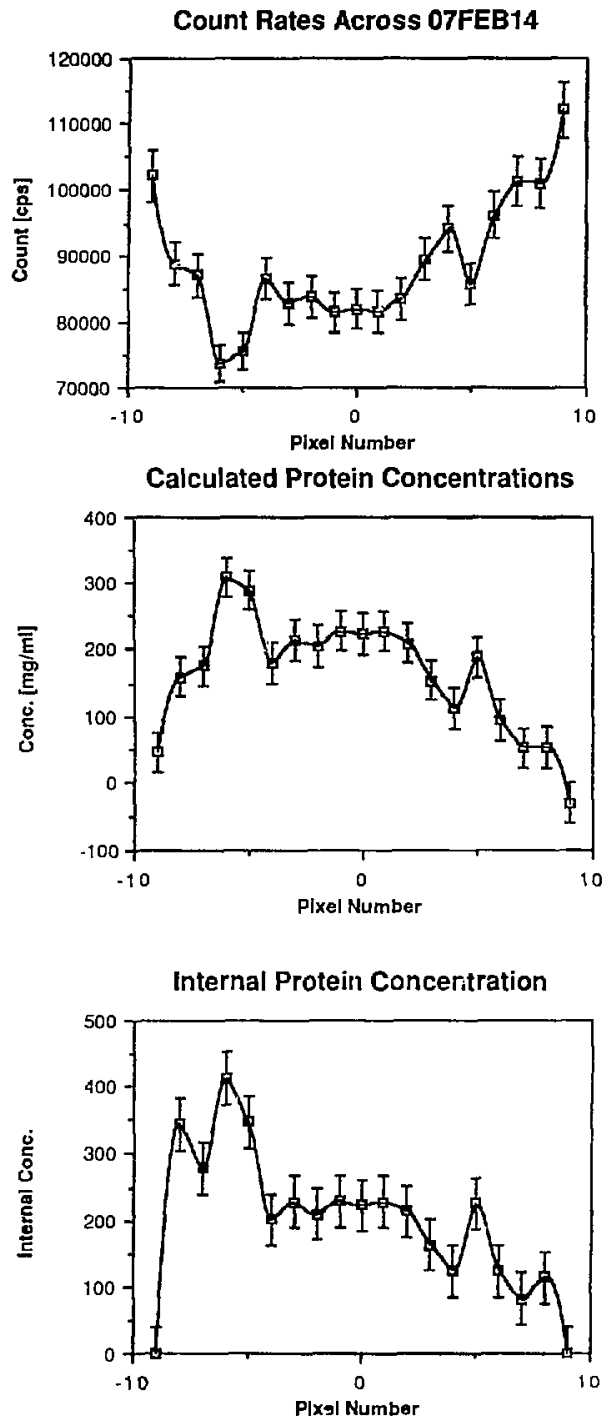

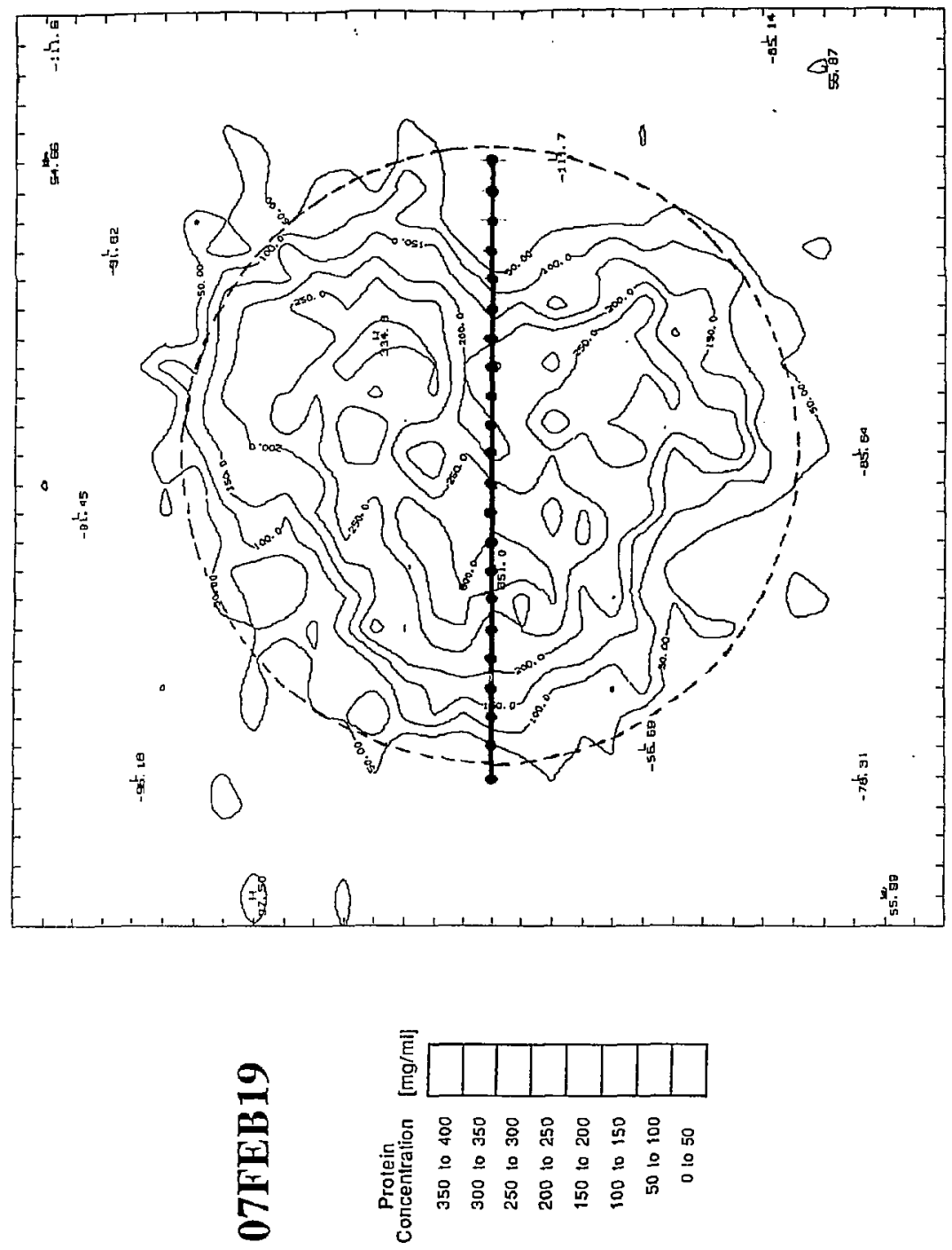
Count Rates Across 07FEB19
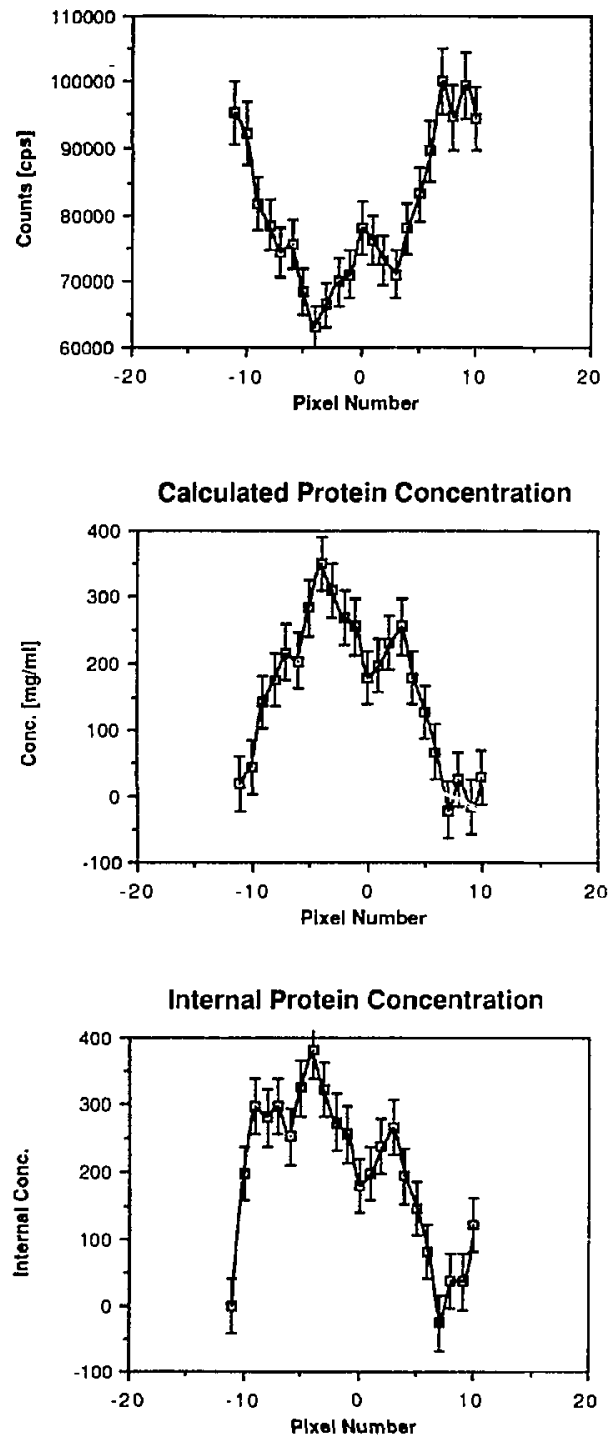
Uniform Spherical Model with Statistical

Fluctuations Applied

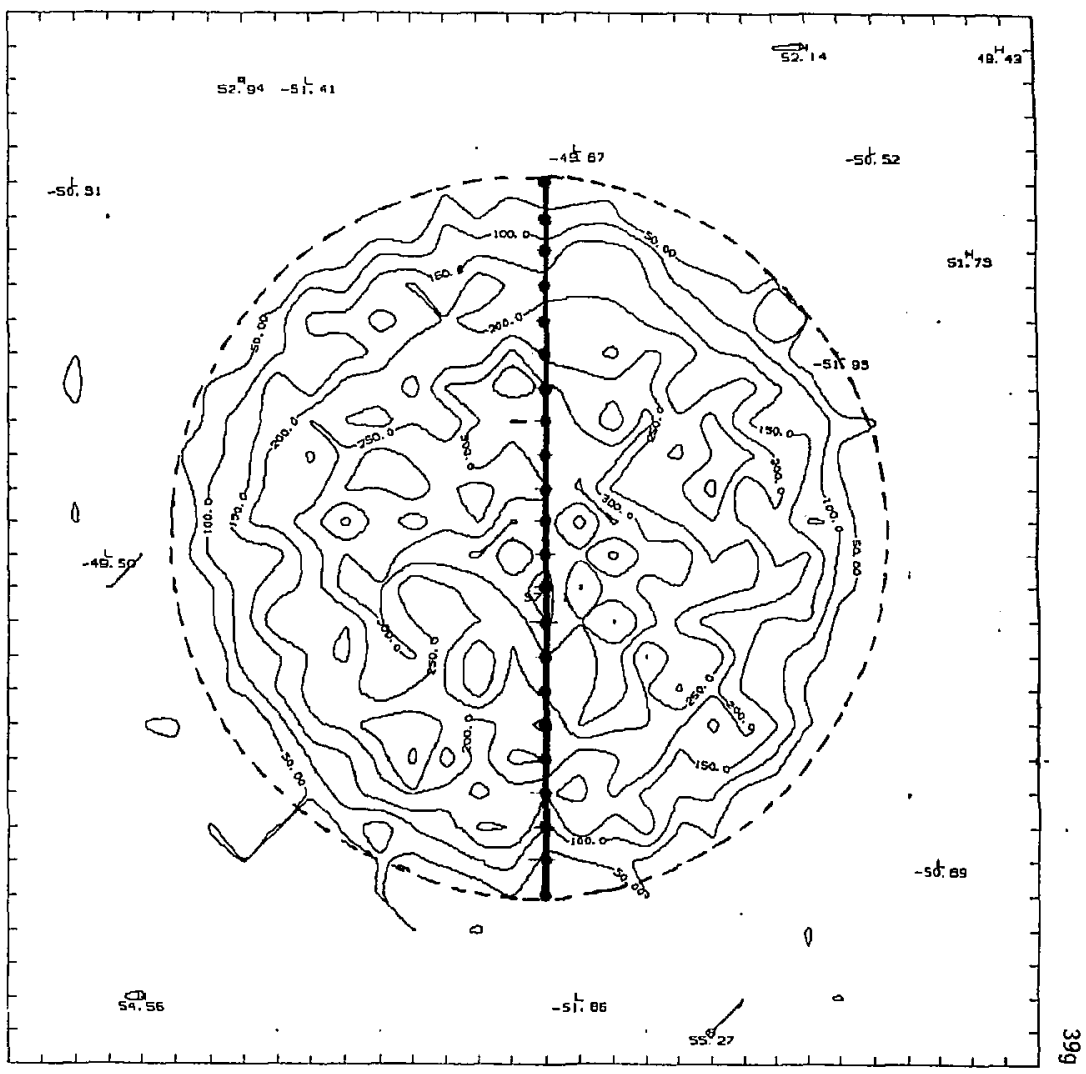




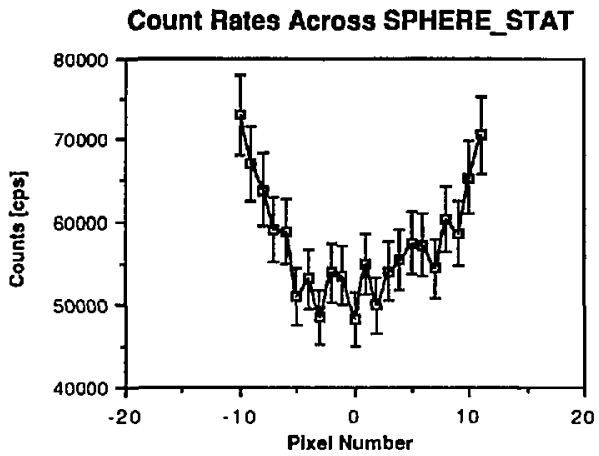

Calculated Protein Concentration
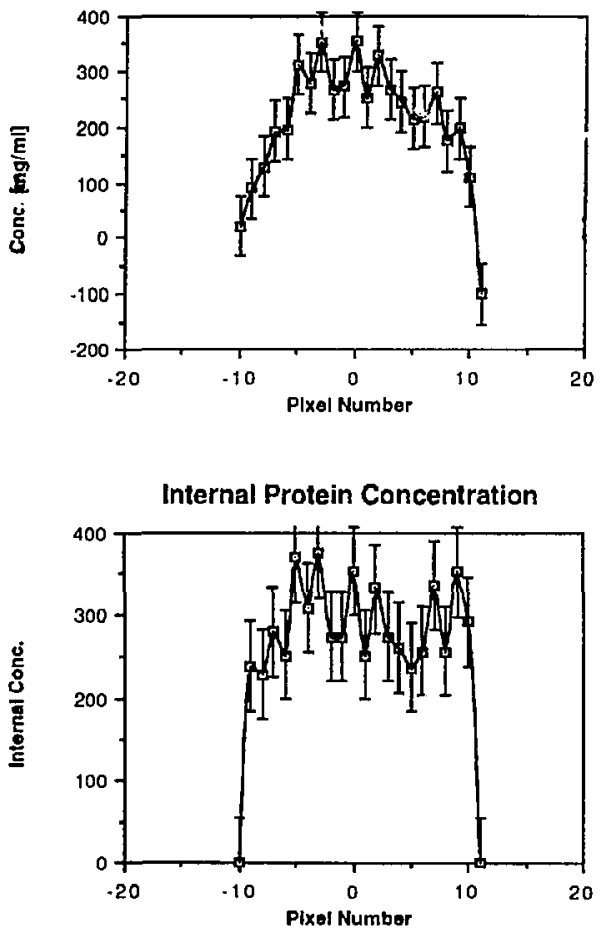
TABLE 5.2

\begin{tabular}{|c|c|c|c|c|}
\hline Quantity & O6FEB20 & O7FEB14 & O7FEB19 & SPHERE_STAT \\
\hline Maximum Internal Count & 417 & 413 & 380 & 375 \\
\hline Minimum Internal Count & 165 & 124 & 179 & 237 \\
\hline Pange of Internal Counts & 252 & 289 & 201 & 138 \\
\hline
\end{tabular}

We notice that the range of internal variation in the real granules is much greater than that of the speherical model. More significant, though, a comparison of the radial distribution of protein along the line of the profile through the real granules and that for the spherical model shows a distinct difference. It is most pronounced in 06FEB20 and 07FEB14. In each of these profiles, the maximum concentrations occur towards the outer edses of the granule. In the profile of 07FEB 19, the maximum is closer to the center. In the case of SPHERE_STAT, as we expect, the conceitration appears almost uniform across most of the profile.

\section{RESULTS}

My numerical values for protein concentrations within the granules compare favorably with values measured by other methods. Estimates ${ }^{[14]}$ made by chemical analysis on a large sample of isolated granules and theoretical consoderations show that the average concentration of protein in a rat granule is $135-270 \mathrm{mg} / \mathrm{ml}$, and that the theoretical upper limit on protein concentrations is $400 \mathrm{mg} / \mathrm{ml}$. My value for the average concentration was $272 \pm 41 \mathrm{mg} / \mathrm{ml}$, and the highest concentrations I saw inside the granules were $417 \pm 39$ and 
$413 \pm 30$. Our values agree to well within the uncertainties in either set of measurements.

In trying to determine the internal organization of the granules, we can see two things. Certainly, the distribution of protein within the granules is not uniform. There are small "islands" of high concentration. Also, the most dense regions are not at the center of the granules. Both of these obseryations suggest some sort of non-uniform organization for the storage of protein in the granules. 


\section{Conclusion}

The nost significant result we obtained was a demonstration of the ability to image fresh, wet, and unaltered biological specimens with a combination of high spatial resolution and elemental sensitivity. Although previous accomplishments had been made imaging fixed specimens in a humid environment so as to prevent dehydration, this is the first time a fresh, unfixed biological specimen, kept in an aqueous environment capable of sustaining life, has been imaged with a scanning $\mathrm{x}$-ray microscope. We obtained biologically interesting data as well, and although there is no clear morphological detail expressed, we can at least see that the notion that these granules are uniform spheres of protein is incorrect.

The scanning transmission $x$-ray microscope fills a unique gap in our ability to study the microscopic world. Countless applications to studying dynamic processes on a sub-cellular level can be found, but several problems must be overcome to fully realize this potential. One problem that immediately comes to mind is the lack of three dimensional information. If we were able to take scans at two or more angles, we could unfold a great deal more information from the data. Another problem is the large degree of uncertainty in the data due to the widely varying count rates. A way to avoid this is to simultaneously monitor the incident beam intensity and the transmitted flux through the sample. This way, the count rate for each pixel can be normalized to the incident bearn intensity, and all of the variation seen would come from changes in absorption in the object. There were plans to do this at X-17T, but it didn't work out.

This experiment has just been an early step in a potentially long and fuitful series of 
applications of the technique of scanning $x$-ray microscopy to the study of biological specimens. Some of the experiements that will soon be possible include a careful comparison of $x$-ray microscope images with electron microscope images of the same specimens. We will also be able to study some of the effects of radiation dosage on a microscopic scale, by doing repeated scans of the same object. By using the natural contrast mechanisms of biological materials at soft $x$-ray wavelengths, we can map out specific elemental distributions within unaltered specimens. We are working now on the development of a "circulating" wet cell, in which whole, living cells could be held. The fluid in which the cells are immersed will be circulated through the wet cell from a reservoir, allowing continuous oxegenation and environmental control. A wet cell such as this would allow the experimenter to stimulate a response from living cells and observe dynamical processes with extremely high resolution. 


\section{References}

[1] Howells, M; et al; 'Soft X-Ray Microscopy", Physics Today, August 1985.

[2] ibid

[3] Vladimirsky, Y; et al.; Proceedings of the Conference on Soft X-Ray Imaging, Berlin, December 1986 . To be published.

[4] Henke, B.; et al.; Atomic Data and Nuclear Data Tables.; Academic Press, vol 27, number 1, January 1982.

[5] Ho, J; Rothman, S; "Protein Concentration in the Pancreatic Zymogen Granule". Biochimica et Biophysica Acta; 755;(1983), pp 457-486

[6] Winick, H; et al; "Wiggler and Undulator Magnets", Physics Today., May 1981.

[7] Hecht, E; Zajac, A; Oprics;Addison Wesley Publishing Co.; Reading, Mass.; 1979.

[8] ibid

[9] Ermak, T.; Rothman, S; "Internal Organization of the Zymogen Granule: Formation of the Reticular Structure in Vitro".Joumal of Ultrastructure Research; 64;98-113 (1978)

[10] Kenney, J.; PHD Dissertation., Department of Physics, SUNY at Stony Brook.

[11] Ho, J; Rothman, S.

[12] Personal Communication with Chris Frieber, Center for X-ray Optics

[13] Ho,J; Rothman,S.

[14] ibid 